\title{
Semiclassical states of nonlinear Dirac equations with degenerate potential
}

\author{
Xu Zhang ${ }^{1} \cdot$ Zhi-Qiang Wang $^{2,3}$
}

Received: 20 December 2018 / Accepted: 26 March 2019 / Published online: 4 April 2019

(c) Fondazione Annali di Matematica Pura ed Applicata and Springer-Verlag GmbH Germany, part of Springer Nature 2019

\section{Abstract}

In this paper, we study the following nonlinear Dirac equation

$$
-i \varepsilon \alpha \cdot \nabla u+a \beta u+V(x) u=|u|^{p-2} u, x \in \mathbb{R}^{3}, \quad \text { for } u \in H^{1}\left(\mathbb{R}^{3}, \mathbb{C}^{4}\right),
$$

where $p \in(2,3), a>0$ is a constant, $\alpha=\left(\alpha_{1}, \alpha_{2}, \alpha_{3}\right), \alpha_{1}, \alpha_{2}, \alpha_{3}$ and $\beta$ are $4 \times 4$ Pauli-Dirac matrices. Our investigation focuses on the case in which $|V(x)|$ may approach $a$ as $|x| \rightarrow \infty$. This is a degenerate case as most works in the literature assume a strict gap condition $\sup _{x \in \mathbb{R}^{3}}|V(x)|<a$, which is a key condition used in setting up an infinitely dimensional topological linking structure as well as in dealing with the compactness issues of the variational formulation. Under the assumption that $V$ has a local trapping potential well, for $\varepsilon>0$ small, we construct bound state solutions concentrating around the local minimum points of $V$. As a consequence we construct an infinite sequence of localized bound state solutions as $\varepsilon \rightarrow 0$.

Keywords Dirac equation $\cdot$ Degenerate potential $\cdot$ Semiclassical states $\cdot$ Concentration

Mathematics Subject Classification 35Q40 - 49J35

\section{Introduction and main results}

In this paper, we study the existence of semiclassical states of the following stationary Dirac equation

Dedicated to Antonio Ambrosetti on the occasion of his 75th birthday.

$\bowtie$ Zhi-Qiang Wang

zhi-qiang.wang@usu.edu

$\mathrm{Xu}$ Zhang

darkblue1121@163.com

1 School of Mathematics and Statistics, Central South University, Changsha 410083, China

2 School of Mathematics and Informatics, Fujian Normal University, Fuzhou 350117, China

3 Department of Mathematics and Statistics, Utah State University, Logan, UT 84322, USA 


$$
-i \varepsilon \sum_{k=1}^{3} \alpha_{k} \partial_{k} u+a \beta u+V(x) u=|u|^{p-2} u, x \in \mathbb{R}^{3}, \text { for } u \in H^{1}\left(\mathbb{R}^{3}, \mathbb{C}^{4}\right) \text {. }
$$

Here, $\varepsilon>0$ is a small parameter, $a>0$ is a constant, $p \in(2,3), \partial_{k}=\partial / \partial x_{k}, V: \mathbb{R}^{3} \rightarrow \mathbb{R}$, $\alpha_{1}, \alpha_{2}, \alpha_{3}$ and $\beta$ are $4 \times 4$ Pauli-Dirac matrices:

$$
\beta=\left(\begin{array}{cc}
I & 0 \\
0 & -I
\end{array}\right), \quad \alpha_{k}=\left(\begin{array}{cc}
0 & \sigma_{k} \\
\sigma_{k} & 0
\end{array}\right), \quad k=1,2,3,
$$

with

$$
\sigma_{1}=\left(\begin{array}{ll}
0 & 1 \\
1 & 0
\end{array}\right), \quad \sigma_{2}=\left(\begin{array}{cc}
0 & -i \\
i & 0
\end{array}\right), \quad \sigma_{3}=\left(\begin{array}{cc}
1 & 0 \\
0 & -1
\end{array}\right) .
$$

Equation (D) or more general versions of the form

$$
-i \hbar \sum_{k=1}^{3} \alpha_{k} \partial_{k} u+a \beta u+V(x) u=F_{u}(x, u)
$$

are related to time-dependent nonlinear Dirac equation

$$
-i \hbar \partial_{t} \psi=i c \hbar \sum_{k=1}^{3} \alpha_{k} \partial_{k} \psi-m c^{2} \beta \psi-M(x) \psi+F_{\psi}(x, \psi)
$$

when one seeks stationary solutions of the form $\psi(t, x)=e^{\frac{i \mu t}{\hbar}} u(x)$ under the condition $F\left(x, e^{i \theta} \psi\right)=F(x, \psi)$ for all $\theta \in[0,2 \pi]$. In Eq. (1.2), $\hbar$ is the Planck constant, $m$ the mass, $c$ the light speed. The external fields in (1.2) arise in models of mathematical physics such as in nonlinear optics. Various nonlinearities appear in models for unified field theories (see $[24,25]$ and references therein).

Mathematical work on nonlinear Dirac-type equations like the above has been studied in recent years, and the existence and multiplicity results for such type equations have been discussed in many papers under different assumptions on the potential and nonlinearity (see [3-5,8,14-19,21-23,29] and references therein). One key condition that has been assumed in these studies is the strict gap condition, namely,

$$
\sup _{\mathbb{R}^{3}}|V(x)|<a .
$$

This is a condition that allows to make use of the spectral decomposition of the linear Dirac operator $-i \sum_{k=1}^{3} \alpha_{k} \partial_{k}+a \beta$ and to set up the abstract space decomposition for an infinitely dimensional topological linking structure as well as to deal with the compactness issues involved. This condition seems to be used in all the papers such as $[3,8,29]$ using ODE approach to obtain special solutions with symmetry reductions, [22,23] using global variational methods for autonomous cases, [4,5,14-19,21] using global variational methods for cases with various external potentials. This is the motivation of our current paper, and we propose a variational framework to tackle the case in which $|V(x)|$ may approach $a$ as $|x| \rightarrow \infty$. More precisely we assume that the potential function $V$ possesses a locally trapping region, $|V(x)|<a$, but $|V(x)|$ may approach $a$ as $|x|$ tends to infinity, and we will find nontrivial solutions concentrating around the trapping region.

To state our main result, we make the following assumptions. 
$\left(\mathrm{V}_{1}\right) V \in C^{1}\left(\mathbb{R}^{3}, \mathbb{R}\right)$, for constants $\tau \in(0,2)$ and $\gamma \in(0,+\infty)$

$$
a-|V(x)| \geq \frac{\gamma}{1+|x|^{\tau}} .
$$

$\left(\mathrm{V}_{2}\right)$ There is a bounded domain $\Lambda \subset \mathbb{R}^{3}$ with smooth boundary $\partial \Lambda$ such that

$$
\vec{n}(x) * \nabla V(x)>0, \text { for any } x \in \partial \Lambda,
$$

where $\vec{n}(x)$ denotes the unit outward normal vector to $\partial \Lambda$ at $x$ and $*$ denotes the inner product in $\mathbb{R}^{3}$.

Note that $\left(\mathrm{V}_{2}\right)$ is satisfied if $V$ has an isolated local minimum set, i.e., $V$ has a locally trapping potential well. Under assumption $\left(\mathrm{V}_{2}\right)$, the set of critical points of $V$

$$
\mathcal{V}=\{x \in \Lambda: \nabla V(x)=0\} \neq \emptyset,
$$

and $\mathcal{V}$ is a compact subset of $\Lambda$. Without loss of generality, we will assume $0 \in \mathcal{V}$. For any set $\Omega \subset \mathbb{R}^{3}, \delta>0$, we define

$$
\Omega^{\delta}=\left\{x \in \mathbb{R}^{3}: \operatorname{dist}(x, \Omega):=\inf _{y \in \Omega}|x-y|<\delta\right\} .
$$

The main result of this paper is as follows.

Theorem 1.1 Suppose that $p \in(2,3)$ and that $\left(\mathrm{V}_{1}\right),\left(\mathrm{V}_{2}\right)$ hold. Then there exists $\varepsilon_{0}>0$ such that if $0<\varepsilon<\varepsilon_{0}$, (D) has a nontrivial solution $u_{\varepsilon}$, satisfying that for any $\delta>0$, there exist $c=c(\delta)>0$ and $C=C(\delta)>0$ such that

$$
\left|u_{\varepsilon}(x)\right| \leq C \exp \left(-c\left(\frac{\operatorname{dist}\left(x, \mathcal{V}^{\delta}\right)}{\varepsilon}\right)^{\frac{2-\tau}{2}}\right) .
$$

The above theorem is easily generalized to more general cases replacing $|u|^{q-2} u$ by $F_{u}(u)$ with $F$ and its derivative $F_{u}$ satisfying some superlinear-type conditions. In particular, it does not need $F$ to be an even function of $u$. However, if $F$ is even in $u$ like the case $|u|^{q-2} u$ we can establish multiple localized solutions concentrating at the local minimum sets $V$ or maximum sets of $K$. In fact, an infinite sequence of such solutions can be constructed as $\varepsilon \rightarrow 0$. We state a multiplicity result as follows.

Theorem 1.2 Suppose that $p \in(2,3)$. When $\left(\mathrm{V}_{1}\right),\left(\mathrm{V}_{2}\right)$ hold, for any positive integer $N$, there exists $\varepsilon_{N}>0$ such that if $0<\varepsilon<\varepsilon_{N}$, (D) has $N$ pairs of nontrivial solutions satisfying the localization property in Theorem 1.1 .

To compare our results above with the existing work in the literature we first elaborate the state of the art of the current studies here. The novelty in our result above is that we can treat the cases where $V(x)$ may approach $a$ or $-a$ as $|x| \rightarrow \infty$ and that we give the existence and infinity multiplicity of solutions concentrating near a local minimum of the potential functions, while the known work in the literature requires lim $\sup _{|x| \rightarrow \infty}|V(x)|<a$ and giving solutions with an upper bound of the number of solutions depending upon both local and global behaviors of the potentials. First, it is well known that there have been many results that relate to the existence and concentration phenomenon of semiclassical states for Schrödinger equations. However, given the classical work on the existence theory of solutions like in [1,3,8,22,29] (and the survey paper [23] for more references therein), such results on localized concentrating semiclassical states for Dirac equations are relatively new and few. In [15], Ding considered (1.1) with $V(x) \equiv 0$ and $F_{u}(x, u)=P(x)|u|^{p-2} u$, 
$p \in(2,3)$ and showed the existence of a ground state solution of the equation for small $\hbar$, which concentrates around the global maximum points of $P(x)$ as $\hbar \rightarrow 0$. This result was later generalized to the case

$$
V(x) \not \equiv 0, \quad \min _{x \in \mathbb{R}^{3}} V(x)<\lim _{|x| \rightarrow+\infty} V(x),
$$

with nonlinearity of the form $F_{u}(x, u)=g(|u|) u, F_{u}(x, u)=P(x) g(|u|) u$, and $F_{u}(x, u)=$ $P(x)(g(|u|)+|u|) u$ in $[16,18]$. Condition (1.4) resembles the classical global condition of Rabinowitz [32] for nonlinear Schrödinger equations, and it is natural to ask whether this can be replaced by a local analogue. In [21], Ding and $\mathrm{Xu}$ weakened the assumptions on $V(x)$ to the following local version: there is a bounded domain $\Lambda \subset \mathbb{R}^{3}$ such that

$$
\min _{x \in \bar{\Lambda}} V(x)<\min _{x \in \partial \Lambda} V(x)
$$

and they established the existence and concentration of a localized solution concentrating in $\Lambda$ when $F_{u}(x, u)=g(|u|) u$ possesses a superlinear or a asymptotically linear growth at infinity. However, in almost all the results involving semiclassical states of Dirac equations, the strict gap condition $|V|_{\infty}<a$ is required, and the only exception is [18], in Theorem 2.5 of which, the authors assumed that $-a \leq V(x) \leq 0$ and $-a<\liminf _{|x| \rightarrow \infty} V(x)$. This hypothesis ensures that the corresponding functional possesses the linking structure, but the solutions found in [18] in fact tend to zero as $\varepsilon \rightarrow 0$, seemingly not of localized type. If $|V(x)| \rightarrow a$ as $|x| \rightarrow \infty$ the variational functional no longer possesses a linking structure. As far as we know, the question on whether there exist nontrivial solutions in this case remains open. We address this question in the present paper, and our Theorem 1.1 gives an affirmative answer to this question under some slow-decay conditions on the potentials. While giving stronger result of the existence of nontrivial localized solutions, we also give the multiplicity results in Theorem 1.2 proving an infinite sequence of concentrating solutions at a local minimum of $V$. Moreover, the system with competing potentials is also considered in Sect. 5.

Regarding the methods involved, for the existence of solutions given in the above references, the solutions are constructed by linking theorem or mountain pass theorem for the reduced functional at critical levels of the associated variational problems where a compactness condition (such as the (PS) condition) holds when $\varepsilon$ is small. However, when $|V(x)|$ tends to $a$ at infinity, we do not have a linking structure, and the boundedness of the (PS) sequence is difficult to get directly. In addition, when we construct multiple solutions, the solutions will be at energy levels where the (PS) condition fails to hold. This situation is somewhat similar to that for nonlinear Schrödinger equations with vanishing potentials (see $[2,30]$ for example), in which case, we usually consider the variational problem in some weighted Sobolev spaces. However, $|V(x)|$ may tend to $a$ at infinity and hence we have to make use of the spectral decomposition of the linear Dirac operator $-i \sum_{k=1}^{3} \alpha_{k} \partial_{k}+a \beta$. This implies that the desired space in which we consider the problem is $E=E^{+} \oplus E^{-}$, and we cannot consider the variational problem in some other spaces with norm weaker than $E$. Moreover, the method of truncating $V$ is hardly to work either due to a lack of a priori estimates of decay rates of solutions. Instead, we consider the problem in the original space $E=E^{+} \oplus E^{-}$and we make use of an idea of penalization similar to that used in $[7,10]$ in the energy functional by adding a penalized functional term $Q_{\varepsilon}$. In this way, we can ensure the existence of a linking structure. Then we also modify the nonlinearity by a similar approach as that in $[12,13]$. Such a modification, together with the construction of the penalization $Q_{\varepsilon}$, makes the (PS) sequence bounded and also converge strongly in E. Finally to return 
to the original problem, we make use of some ideas for nonlinear Schrödinger equations, e.g., $[6,7,9,10,12,13,27,32]$, in particular in the recent work of [10] in which for nonlinear Schrödinger equations an infinite sequence of localized nodal solutions was constructed near a local minimum of the potential function. In our recent work [33] we have also used similar idea to deal with the nonlinear Dirac equations under the strict gap condition between $|V(x)|$ and $a$.

The remainder of this paper is organized as follows. In Sect. 2, we derive a variational setting for the problem, introduce the modified functional by a penalization approach and give some preliminary lemmas. In Sect. 3, by using a linking theorem, we show the existence of nontrivial solutions of the modified problems when $\varepsilon$ is small. In Sect. 4 , we give a localization argument to prove that these solutions to the modified problems are in fact solutions of the original problem for $\varepsilon$ small and finish the proof of main Theorem 1.1. In Sect. 5, we give the proof for Theorem 1.2 and finish the paper by sketching the proof for the system with competing potentials.

\section{Preliminaries}

To prove the main results, some preliminaries are firstly in order. First, using the scaling $w(x)=u(\varepsilon x)$, it is easy to see that $u$ is a solution of (D) if and only if $w$ is a solution of

$$
-i \alpha \cdot \nabla w+a \beta w+V(\varepsilon x) w=|w|^{p-2} w .
$$

Therefore, we will mainly focus on this equivalent equation in the remaining part of the paper. For any set $\Omega \subset \mathbb{R}^{3}$ and $\varepsilon>0$, we define

$$
\Omega_{\varepsilon}=\left\{x \in \mathbb{R}^{3}: \varepsilon x \in \Omega\right\} .
$$

We denote by $|\cdot|_{q}$ the usual $L^{q}$ norm and by $\langle\cdot, \cdot\rangle_{2}$ the $L^{2}$ inner product. Let $H_{0}=-i \alpha$. $\nabla+a \beta$ denote the self-adjoint operator on $L^{2}\left(\mathbb{R}^{3}, \mathbb{C}^{4}\right)$ with domain $\mathcal{D}\left(H_{0}\right)=H^{1}\left(\mathbb{R}^{3}, \mathbb{C}^{4}\right)$. It is clear that $\sigma\left(H_{0}\right)=\sigma_{e}\left(H_{0}\right)=\mathbb{R} \backslash(-a, a)$, where $\sigma\left(H_{0}\right)$ and $\sigma_{e}\left(H_{0}\right)$ denote the spectrum and the essential spectrum of $H_{0}$, respectively. Thus the space $L^{2}\left(\mathbb{R}^{3}, \mathbb{C}^{4}\right)$ possesses the orthogonal decomposition:

$$
L^{2}\left(\mathbb{R}^{3}, \mathbb{C}^{4}\right)=L^{+} \oplus L^{-},
$$

such that $H_{0}$ is positive definite in $L^{+}$and negative definite in $L^{-}$. Thus for $u \in L^{2}\left(\mathbb{R}^{3}, \mathbb{C}^{4}\right)$, we could denote by $u=u^{+}+u^{-}$the decomposition of $u$ with $u^{+} \in L^{+}$and $u^{-} \in L^{-}$.

Let $\left|H_{0}\right|$ denote the absolute value of $H_{0}$ and $\left|H_{0}\right|^{\frac{1}{2}}$ denote its square root. We define $E:=\mathcal{D}\left(\left|H_{0}\right|^{\frac{1}{2}}\right)$ endowed with the inner product

$$
\langle u, v\rangle=\left\langle\left|H_{0}\right|^{\frac{1}{2}} u,\left|H_{0}\right|^{\frac{1}{2}} v\right\rangle_{2}
$$

and the induced norm $\|u\|^{2}=\langle u, u\rangle$. From [14] Lemma 7.4, it follows that $E=H^{\frac{1}{2}}\left(\mathbb{R}^{3}, \mathbb{C}^{4}\right)$ and the norm $\|\cdot\|$ is equivalent to the usual norm in $H^{\frac{1}{2}}\left(\mathbb{R}^{3}, \mathbb{C}^{4}\right)$. Hence, $E$ embeds into $L^{q}\left(\mathbb{R}^{3}, \mathbb{C}^{4}\right)$ continuously for $q \in[2,3]$ and into $L_{\text {loc }}^{q}\left(\mathbb{R}^{3}, \mathbb{C}^{4}\right)$ compactly for $q \in[1,3)$. Moreover, since $\sigma\left(H_{0}\right)=\mathbb{R} \backslash(-a, a)$, we have

$$
a|u|_{2}^{2} \leq\|u\|^{2} \text {, for all } u \in E .
$$


It is clear that (see [14] Section 7) $E$ possesses the following decomposition

$$
E=E^{+} \oplus E^{-},
$$

where $E^{+}=E \cap L^{+}$and $E^{-}=E \cap L^{-}$and the sum is orthogonal with respect to both $\langle\cdot, \cdot\rangle_{2}$ and $\langle\cdot, \cdot \cdot\rangle$. Additionally, this decomposition of $E$ induces also a natural decomposition of $L^{q}\left(\mathbb{R}^{3}, \mathbb{C}^{4}\right)$ for $q \in(1,+\infty)$ and we refer the readers to [21] Proposition 2.1 for the proof of the following proposition.

Proposition 2.1 Let $E^{+} \oplus E^{-}$be the decomposition of $E$ according to the positive and negative part of $\sigma\left(H_{0}\right)$. Then, if we set $E^{ \pm}{ }_{q}:=E^{ \pm} \cap L^{q}\left(\mathbb{R}^{3}, \mathbb{C}^{4}\right)$ for $q \in(1,+\infty)$, there holds

$$
L^{q}\left(\mathbb{R}^{3}, \mathbb{C}^{4}\right)=\mathrm{cl}_{q} E_{q}^{+} \oplus \mathrm{cl}_{q} E_{q}^{-},
$$

where $\mathrm{cl}_{q}$ denotes the closure in $L^{q}\left(\mathbb{R}^{3}, \mathbb{C}^{4}\right)$. Moreover, for any $q \in(1,+\infty)$, there exists positive constant $d_{q}$ such that

$$
d_{q}\left|u^{ \pm}\right|_{q} \leq|u|_{q}, \quad u \in E \cap L^{q}\left(\mathbb{R}^{3}, \mathbb{C}^{4}\right) .
$$

According to standard arguments, the functional $J_{\varepsilon}: E \rightarrow \mathbb{R}$ defined by

$$
J_{\varepsilon}(u)=\frac{1}{2}\left(\left\|u^{+}\right\|^{2}-\left\|u^{-}\right\|^{2}\right)+\frac{1}{2} \int_{\mathbb{R}^{3}} V(\varepsilon x)|u|^{2}-\int_{\mathbb{R}^{3}}|u|^{p}
$$

is of class $C^{1}$. For $u, v \in E$, there holds

$$
J_{\varepsilon}^{\prime}(u) v=\operatorname{Re} \int_{\mathbb{R}^{3}}\left(H_{0} u+V(\varepsilon x) u-|u|^{p-2} u\right) \cdot v
$$

with $u \cdot v$ denoting the usual inner product in $\mathbb{C}^{4}$, i.e., $u \cdot v=\sum_{j=1}^{4} u_{j} \bar{v}_{j}$. Hence the critical points of $J_{\varepsilon}$ are weak solutions of ( $\mathrm{D}_{\varepsilon}$ ) (one can find more details in [15]).

As we have mentioned in Sect. 1 , we will not deal with $J_{\varepsilon}$ directly. Instead, we need make use of some penalization approaches such as that used in $[7,10,12,13]$ to modify the functional such that it possesses nontrivial solutions. After constructing solutions of the modified problems we will make these solutions localized, so they are solutions of the original problem for small $\varepsilon$. This part of the work will be done in Sect. 4 .

By $\left(\mathrm{V}_{2}\right)$, we can deduce that there exists $\delta_{1}>0$ such that for any $y \in \Lambda^{\delta_{1}}$, if $B\left(y, \delta_{1}\right) \backslash \bar{\Lambda} \neq$ $\emptyset$, there holds

$$
\inf _{x \in B\left(y, \delta_{1}\right) \backslash \bar{\Lambda}} \nabla V(y) * \nabla \operatorname{dist}(x, \Lambda)>0 .
$$

where $*$ denotes the usual inner product in $\mathbb{R}^{3}, \Lambda^{\delta_{1}}$ is the $\delta_{1}$-neighborhood of $\Lambda$ which we defined in (1.3) and $B(x, r)$ denotes the open ball in $\mathbb{R}^{3}$ with center $x \in \mathbb{R}^{3}$ and radius $r>0$.

According to $\left(\mathrm{V}_{1}\right)$, we can choose $\theta \in(0,1)$ and a big $\widetilde{R}>0$ such that $\Lambda^{\delta_{1}+1} \subset$ $B(0, \widetilde{R} / 2), 2 \gamma /\left(1+\widetilde{R}^{\tau}\right) \leq a$ and

$$
|V(x)| \leq \theta a,|x| \leq \widetilde{R}+1 .
$$

Let $\underset{\zeta}{\tilde{\zeta}} \in C^{\infty}(\mathbb{R},[0,1])$ be a cutoff function such that $\widetilde{\zeta}(t)=0$ if $t \leq 0, \widetilde{\zeta}(t)>0$ if $t>0$ and $\widetilde{\zeta}(t)=1$ if $t \geq 1$. Then we set

$$
\tilde{\chi}(x)=\widetilde{\zeta}(|x|-\widetilde{R}), \quad x \in \mathbb{R}^{3} .
$$


Fixing $\phi(x)=\frac{1}{1+|x|^{4}}$, we define $\xi, \widehat{\xi}: \mathbb{R}^{3} \times \mathbb{R} \rightarrow \mathbb{R}$ by

$$
\xi(x, t)= \begin{cases}0, & t \leq \phi(x) ; \\ \frac{1}{\phi(x)}(t-\phi(x))^{2}, & \phi(x)<t<2 \phi(x) ; \quad \widehat{\xi}(x, t)=\int_{-\infty}^{t} \xi(x, s) \mathrm{d} s \\ 2 t-3 \phi(x), & t \geq 2 \phi(x),\end{cases}
$$

and define the penalized functional $Q_{\varepsilon}: E \rightarrow \mathbb{R}$ by

$$
Q_{\varepsilon}(u)=\frac{1}{8} \int_{\mathbb{R}^{3}} \tilde{\chi}(\varepsilon x) V(\varepsilon x) \widehat{\xi}(x,|u|) .
$$

Using the definition of $\xi$ and $\widehat{\xi}$, it is easy to verify that $Q_{\varepsilon} \in C^{1}(E, \mathbb{R})$,

$$
Q_{\varepsilon}^{\prime}(u) \varphi=\frac{1}{8} \operatorname{Re} \int_{\mathbb{R}^{3}} \tilde{\chi}(\varepsilon x) V(\varepsilon x) \widetilde{\xi}(x,|u|) u \cdot \varphi \text { for any } \varphi \in C_{0}^{\infty}\left(\mathbb{R}^{3}, \mathbb{C}^{4}\right),
$$

where $\tilde{\xi}(x, t) \in C^{1}\left(\mathbb{R}^{3} \times \mathbb{R},[0,2]\right)$,

$$
\widetilde{\xi}(x, t):=\frac{1}{t} \xi(x, t)= \begin{cases}0, & t \leq \phi(x) \\ \frac{(t-\phi(x))^{2}}{t \phi(x)}, & \phi(x)<t<2 \phi(x) ; \\ \frac{2 t-3 \phi(x)}{t}, & t \geq 2 \phi(x) .\end{cases}
$$

Moreover, for $u_{n} \rightarrow u$ weakly in $E$, there holds

$$
Q_{\varepsilon}^{\prime}\left(u_{n}\right) \varphi \rightarrow Q_{\varepsilon}^{\prime}(u) \varphi \text { for any } \varphi \in C_{0}^{\infty}\left(\mathbb{R}^{3}, \mathbb{C}^{4}\right) .
$$

Remark 2.2 The penalized functional $Q_{\varepsilon}$ behaves like $\frac{1}{8} \int_{\mathbb{R}^{3}} \tilde{\chi}(\varepsilon x) V(\varepsilon x)(|u|-\phi)_{+}^{2}$, which is modified to possess more smoothness at $u=\phi$. It is a local version penalization in the sense of integral such that (2.8) holds. In fact, this property is important to our arguments and is required in the infinite-dimensional linking theorem for the strong indefinite problem, by which we will construct multiple critical levels of the problem in Sects. 3 and 5. It also should be noted that although our $Q_{\varepsilon}$ and the penalization used in [7,10] have similar forms, they play different roles. More precisely, our $Q_{\varepsilon}$ ensures the linking structure of the functional, while the penalization in $[7,10]$ makes the bounded Palais-Smale sequence to be relatively compact.

Let $\zeta \in C^{\infty}(\mathbb{R},[0,1])$ be a cutoff function such that $\zeta^{\prime}(t) \geq 0$ for every $t \in \mathbb{R}, \zeta(t)=0$ if $t \leq 0,0<\zeta(t)<1$ if $0<t<\delta_{1}$ and $\zeta(t)=1$ if $t \geq \delta_{1}$. And we set $\chi(x)=\zeta(\operatorname{dist}(x, \Lambda))$ and $g \in C\left(\mathbb{R}^{3} \times \mathbb{R}^{+}, \mathbb{R}\right)$ :

$$
g(x, t)=\min \left\{t^{p-2}, \phi(x)\right\} .
$$

Define

$$
f_{\varepsilon}(x, t)=(1-\chi(\varepsilon x)) t^{p-2}+\chi(\varepsilon x) g(x, t), \quad F_{\varepsilon}(x, t)=\int_{0}^{t} f_{\varepsilon}(x, s) s \mathrm{~d} s .
$$

We can verify that $f$ is a Caratheodory function which satisfies:

$$
\left(f_{1}\right) 0 \leq f_{\varepsilon}(x, t) \leq t^{p-2} \text { for every }(x, t) \in \mathbb{R}^{3} \times \mathbb{R}^{+} \text {; }
$$


$\left(f_{2}\right)$ for $(x, t) \in \mathbb{R}^{3} \times \mathbb{R}^{+}$,

$$
F_{\varepsilon}(x, t)=(1-\chi(\varepsilon x)) \frac{t^{p}}{p}+\chi(\varepsilon x) G(x, t),
$$

where $G(x, t)=\int_{0}^{t} g(x, s) s \mathrm{~d} s$ satisfies $g(x, t) t^{2}-2 G(x, t) \geq 0$.

Now we define the modified functional $\Phi_{\varepsilon}: E \rightarrow \mathbb{R}$,

$$
\Phi_{\varepsilon}(u)=\frac{1}{2}\left(\left\|u^{+}\right\|^{2}-\left\|u^{-}\right\|^{2}\right)+\frac{1}{2} \int_{\mathbb{R}^{3}} V(\varepsilon x)|u|^{2}-Q_{\varepsilon}(u)-\int_{\mathbb{R}^{3}} F_{\varepsilon}(x,|u|) .
$$

According to (2.6), $\Phi_{\varepsilon}$ is of class $C^{1}$. Moreover, for $u, v \in E$, there holds

$$
\Phi_{\varepsilon}^{\prime}(u) v=\operatorname{Re} \int_{\mathbb{R}^{3}}\left(H_{0} u+V(\varepsilon x) u-\frac{1}{8} \tilde{\chi}(\varepsilon x) V(\varepsilon x) \tilde{\xi}(x,|u|) u-f_{\varepsilon}(x,|u|) u\right) \cdot v,
$$

and the critical points correspond to weak solutions of

$$
-i \alpha \cdot \nabla u+a \beta u+V(\varepsilon x) u-\frac{1}{8} \tilde{\chi}(\varepsilon x) V(\varepsilon x) \tilde{\xi}(x,|u|) u=f_{\varepsilon}(x,|u|) u .
$$

Lemma 2.3 For small $\varepsilon_{0}>0$ and $\varepsilon \in\left(0, \varepsilon_{0}\right)$, let $\left\{u_{n}\right\}$ be a Palais-Smale sequence of the functional $\Phi_{\varepsilon}$ at level $c$. Then $u_{n}$ converge strongly in $E$.

Proof Our first step is to show the boundedness of $\left\{u_{n}\right\}$ in $E$.

$$
\begin{aligned}
c+\left\|u_{n}\right\| \geq \Phi_{\varepsilon}\left(u_{n}\right)-\frac{1}{2} \Phi_{\varepsilon}^{\prime}\left(u_{n}\right) u_{n}= & \int_{\mathbb{R}^{3}} \frac{1}{2} f_{\varepsilon}\left(x,\left|u_{n}\right|\right)\left|u_{n}\right|^{2}-F_{\varepsilon}\left(x,\left|u_{n}\right|\right) \\
& +\frac{1}{2} Q_{\varepsilon}^{\prime}\left(u_{n}\right) u_{n}-Q_{\varepsilon}\left(u_{n}\right) .
\end{aligned}
$$

By the definition of $Q_{\varepsilon}$ and (2.5)-(2.7), we have

$$
\frac{1}{2} Q_{\varepsilon}^{\prime}\left(u_{n}\right) u_{n}-Q_{\varepsilon}\left(u_{n}\right) \geq-a \int_{\mathbb{R}^{3}} \tilde{\chi}(\varepsilon x)\left|u_{n}\right| \phi \geq-C\left\|u_{n}\right\| .
$$

Combining this with $\left(f_{2}\right)$, we have

$$
\begin{aligned}
C\left(1+\left\|u_{n}\right\|\right) & \geq \int_{\mathbb{R}^{3}} \frac{1}{2} f_{\varepsilon}\left(x,\left|u_{n}\right|\right)\left|u_{n}\right|^{2}-F_{\varepsilon}\left(x,\left|u_{n}\right|\right) \\
& \geq \frac{p-2}{2 p} \int_{\mathbb{R}^{3}}(1-\chi(\varepsilon x))\left|u_{n}\right|^{p} .
\end{aligned}
$$

On the other hand, $o(1)\left\|u_{n}\right\|=\Phi_{\varepsilon}^{\prime}\left(u_{n}\right)\left(u_{n}^{+}-u_{n}^{-}\right)$, and hence

$$
\begin{aligned}
o(1)\left\|u_{n}\right\|= & \left\|u_{n}\right\|^{2}+\operatorname{Re} \int_{\mathbb{R}^{3}} V(\varepsilon x) u_{n} \cdot\left(u_{n}^{+}-u_{n}^{-}\right)-f_{\varepsilon}\left(x,\left|u_{n}\right|\right) u_{n} \cdot\left(u_{n}^{+}-u_{n}^{-}\right) \\
& -\frac{1}{8} \operatorname{Re} \int_{\mathbb{R}^{3}} \tilde{\chi}(\varepsilon x) V(\varepsilon x) \widetilde{\xi}\left(x,\left|u_{n}\right|\right) u_{n} \cdot\left(u_{n}^{+}-u_{n}^{-}\right) \\
= & \left\|u_{n}\right\|^{2}+\int_{\mathbb{R}^{3}}\left(V(\varepsilon x)-\frac{1}{8} \tilde{\chi}(\varepsilon x) V(\varepsilon x) \tilde{\xi}\left(x,\left|u_{n}\right|\right)\right)\left(\left|u_{n}^{+}\right|^{2}-\left|u_{n}^{-}\right|^{2}\right) \\
& -\operatorname{Re} \int_{\mathbb{R}^{3}}\left(\chi(\varepsilon x) g\left(x,\left|u_{n}\right|\right) u_{n}+(1-\chi(\varepsilon x))\left|u_{n}\right|^{p-2} u_{n}\right) \cdot\left(u_{n}^{+}-u_{n}^{-}\right) .
\end{aligned}
$$


To estimate the second term in the above equality, using (2.4) and $\widetilde{\xi} \in[0,2]$, it is easy to verify that

$$
\begin{aligned}
& \int_{\mathbb{R}^{3}} V(\varepsilon x)\left|u_{n}^{+}\right|^{2}-\frac{1}{8} \tilde{\chi}(\varepsilon x) V(\varepsilon x) \tilde{\xi}\left(x,\left|u_{n}\right|\right)\left|u_{n}^{+}\right|^{2} \\
& =\int_{\mathbb{R}^{3}}(1-\tilde{\chi}(\varepsilon x)) V(\varepsilon x)\left|u_{n}^{+}\right|^{2}+\left(1-\frac{1}{8} \widetilde{\xi}\left(x,\left|u_{n}\right|\right)\right) \tilde{\chi}(\varepsilon x) V(\varepsilon x)\left|u_{n}^{+}\right|^{2} \\
& \quad \geq \int_{\mathbb{R}^{3}}-\theta a(1-\tilde{\chi}(\varepsilon x))\left|u_{n}^{+}\right|^{2}+\left(1-\frac{1}{8} \widetilde{\xi}\left(x,\left|u_{n}\right|\right)\right) \tilde{\chi}(\varepsilon x) V_{-}(\varepsilon x)\left|u_{n}^{+}\right|^{2} \\
& \quad+\frac{1}{2} \int_{\mathbb{R}^{3}} \tilde{\chi}(\varepsilon x) V_{+}(\varepsilon x)\left|u_{n}^{+}\right|^{2} .
\end{aligned}
$$

where $V_{+}(x):=\max \{V(x), 0\}$ and $V_{-}(x):=\min \{V(x), 0\}$. Setting $\Omega(n)=\{x \in$ $\left.\mathbb{R}^{3}:\left|u_{n}(x)\right| \geq 3 \phi(x)\right\}$, then by the definition of $\widetilde{\xi}$, there holds

$$
\widetilde{\xi}\left(x,\left|u_{n}(x)\right|\right) \geq 1, \quad x \in \Omega(n) .
$$

From this, the facts $\left|u_{n}(x)\right| \leq 3 \phi(x)$ for $x \in \mathbb{R}^{3} \backslash \Omega(n)$ and Young's inequality, it follows that

$$
\begin{aligned}
& \int_{\mathbb{R}^{3}}\left(1-\frac{1}{8} \widetilde{\xi}\left(x,\left|u_{n}\right|\right)\right) \tilde{\chi}(\varepsilon x) V_{-}(\varepsilon x)\left|u_{n}^{+}\right|^{2} \\
& \quad \geq \int_{\Omega(n)}\left(1-\frac{1}{8} \widetilde{\xi}\left(x,\left|u_{n}\right|\right)\right) \tilde{\chi}(\varepsilon x) V_{-}(\varepsilon x)\left|u_{n}^{+}\right|^{2}+\int_{\mathbb{R}^{3} \backslash \Omega(n)} \tilde{\chi}(\varepsilon x) V_{-}(\varepsilon x)\left|u_{n}^{+}\right|^{2} \\
& \quad \geq-\frac{7 a}{8} \int_{\Omega(n)} \tilde{\chi}(\varepsilon x)\left|u_{n}^{+}\right|^{2}+\int_{\mathbb{R}^{3} \backslash \Omega(n)} \tilde{\chi}(\varepsilon x) V_{-}(\varepsilon x)\left(\left|u_{n}\right|^{2}+2\left|u_{n}\right|\left|u_{n}^{-}\right|+\left|u_{n}^{-}\right|^{2}\right) \\
& \geq-\frac{7 a}{8} \int_{\Omega(n)} \tilde{\chi}(\varepsilon x)\left|u_{n}^{+}\right|^{2}+\int_{\mathbb{R}^{3} \backslash \Omega(n)} \tilde{\chi}(\varepsilon x) V_{-}(\varepsilon x)\left|u_{n}^{-}\right|^{2}-C\left(\left\|u_{n}^{-}\right\|+1\right),
\end{aligned}
$$

taking which into (2.11), we have

$$
\begin{aligned}
& \int_{\mathbb{R}^{3}} V(\varepsilon x)\left|u_{n}^{+}\right|^{2}-\frac{1}{8} \tilde{\chi}(\varepsilon x) V(\varepsilon x) \tilde{\xi}\left(x,\left|u_{n}\right|\right)\left|u_{n}^{+}\right|^{2} \\
& \geq \int_{\mathbb{R}^{3}}-\theta a(1-\tilde{\chi}(\varepsilon x))\left|u_{n}^{+}\right|^{2}-\frac{7 a}{8} \int_{\Omega(n)} \tilde{\chi}(\varepsilon x)\left|u_{n}^{+}\right|^{2}+\frac{1}{2} \int_{\mathbb{R}^{3}} \tilde{\chi}(\varepsilon x) V_{+}(\varepsilon x)\left|u_{n}^{+}\right|^{2} \\
& \quad+\int_{\mathbb{R}^{3} \backslash \Omega(n)} \tilde{\chi}(\varepsilon x) V_{-}(\varepsilon x)\left|u_{n}^{-}\right|^{2}-C\left(\left\|u_{n}^{-}\right\|+1\right) .
\end{aligned}
$$

Similarly,

$$
\begin{aligned}
& \int_{\mathbb{R}^{3}}-V(\varepsilon x)\left|u_{n}^{-}\right|^{2}+\frac{1}{8} \tilde{\chi}(\varepsilon x) V(\varepsilon x) \widetilde{\xi}\left(x,\left|u_{n}\right|\right)\left|u_{n}^{-}\right|^{2} \\
& \geq \int_{\mathbb{R}^{3}}-\theta a(1-\tilde{\chi}(\varepsilon x))\left|u_{n}^{-}\right|^{2}-\frac{7 a}{8} \int_{\Omega(n)} \tilde{\chi}(\varepsilon x)\left|u_{n}^{-}\right|^{2}-\frac{1}{2} \int_{\mathbb{R}^{3}} \tilde{\chi}(\varepsilon x) V_{-}(\varepsilon x)\left|u_{n}^{-}\right|^{2} \\
& \quad-\int_{\mathbb{R}^{3} \backslash \Omega(n)} \tilde{\chi}(\varepsilon x) V_{+}(\varepsilon x)\left|u_{n}^{+}\right|^{2}-C\left(\left\|u_{n}^{+}\right\|+1\right) .
\end{aligned}
$$


By the two inequalities above and (2.2), we deduce

$$
\begin{aligned}
& \int_{\mathbb{R}^{3}} V(\varepsilon x)\left(\left|u_{n}^{+}\right|^{2}-\left|u_{n}^{-}\right|^{2}\right)-\frac{1}{8} \tilde{\chi}(\varepsilon x) V(\varepsilon x) \widetilde{\xi}\left(x,\left|u_{n}\right|\right)\left(\left|u_{n}^{+}\right|^{2}-\left|u_{n}^{-}\right|^{2}\right) \\
& \quad \geq-\max \left(\theta, \frac{7}{8}\right)\left\|u_{n}\right\|^{2}-C\left(\left\|u_{n}\right\|+1\right) .
\end{aligned}
$$

To estimate the nonlinearity, using (2.9) and the Hölder's inequality, we have

$$
\left.\left|\int_{\mathbb{R}^{3}}(1-\chi(\varepsilon x))\right| u_{n}\right|^{p-2} u_{n} \cdot\left(u_{n}^{+}-u_{n}^{-}\right) \mid \leq C\left(\left\|u_{n}\right\|+\left\|u_{n}\right\|^{\frac{2 p-1}{p}}\right) .
$$

Moreover, it follows from $0 \in \mathcal{V}$ and the fact $g(x, t) \leq \phi(x)$ that

$$
\left|\int_{\mathbb{R}^{3}} \chi(\varepsilon x) g\left(x,\left|u_{n}\right|\right) u_{n} \cdot\left(u_{n}^{+}-u_{n}^{-}\right)\right| \leq C \varepsilon^{4}\left\|u_{n}\right\|^{2} .
$$

Combining the two inequalities above with $\theta \in(0,1),(2.10)$ and (2.12), we obtain that

$$
\min \left\{1-\theta, \frac{1}{8}\right\}\left\|u_{n}\right\|^{2}-C \varepsilon^{4}\left\|u_{n}\right\|^{2} \leq C\left(1+\left\|u_{n}\right\|+\left\|u_{n}\right\|^{\frac{2 p-1}{p}}\right),
$$

which implies the boundedness of $\left\{u_{n}\right\}$ in $E$ for small $\varepsilon_{0}$ and $\varepsilon \in\left(0, \varepsilon_{0}\right)$. Moreover, there holds

$$
\left\|u_{n}\right\| \leq C^{\prime} \text { where } C^{\prime} \text { depends only on } c \text {, but is independent of } \varepsilon \in\left(0, \varepsilon_{0}\right) .
$$

Up to a subsequence, we have $u_{n} \rightarrow u$ weakly in $E, u_{n}^{ \pm} \rightarrow u^{ \pm}$weakly in $E$ and $u_{n}^{ \pm} \rightarrow u^{ \pm}$ strongly in $L_{\text {loc }}^{q}\left(\mathbb{R}^{3}, \mathbb{C}^{4}\right), q \in[1,3)$. Thus from (2.8), it follows that $u$ weakly solves

$$
-i \alpha \cdot \nabla u+a \beta u+V(\varepsilon x) u-\frac{1}{8} \tilde{\chi}(\varepsilon x) V(\varepsilon x) \widetilde{\xi}(x,|u|) u=f_{\varepsilon}(x,|u|) u .
$$

So for $z_{n}=u_{n}-u$, we have $\Phi_{\varepsilon}^{\prime}\left(u_{n}\right)\left(z_{n}^{+}-z_{n}^{-}\right)=o(1)$ and $\Phi_{\varepsilon}^{\prime}(u)\left(z_{n}^{+}-z_{n}^{-}\right)=0$, and hence

$$
\begin{aligned}
o(1)= & \operatorname{Re}\left\langle u_{n}^{+}, z_{n}^{+}\right\rangle+\operatorname{Re}\left\langle u_{n}^{-}, z_{n}^{-}\right\rangle+\operatorname{Re} \int_{\mathbb{R}^{3}} V(\varepsilon x) u_{n} \cdot\left(z_{n}^{+}-z_{n}^{-}\right) \\
& -\operatorname{Re} \int_{\mathbb{R}^{3}} \frac{1}{8} \tilde{\chi}(\varepsilon x) V(\varepsilon x) \tilde{\xi}\left(x,\left|u_{n}\right|\right) u_{n} \cdot\left(z_{n}^{+}-z_{n}^{-}\right)+f_{\varepsilon}\left(x,\left|u_{n}\right|\right) u_{n} \cdot\left(z_{n}^{+}-z_{n}^{-}\right) ; \\
0= & \operatorname{Re}\left\langle u^{+}, z_{n}^{+}\right\rangle+\operatorname{Re}\left\langle u^{-}, z_{n}^{-}\right\rangle+\operatorname{Re} \int_{\mathbb{R}^{3}} V(\varepsilon x) u \cdot\left(z_{n}^{+}-z_{n}^{-}\right) \\
& -\operatorname{Re} \int_{\mathbb{R}^{3}} \frac{1}{8} \tilde{\chi}(\varepsilon x) V(\varepsilon x) \tilde{\xi}(x,|u|) u \cdot\left(z_{n}^{+}-z_{n}^{-}\right)+f_{\varepsilon}(x,|u|) u \cdot\left(z_{n}^{+}-z_{n}^{-}\right) .
\end{aligned}
$$

By the definition of $g(x, t)$, there holds $g(x, t) \leq \phi(x)$, which implies that

$$
\lim _{n \rightarrow \infty} \operatorname{Re} \int_{\mathbb{R}^{3}} f_{\varepsilon}(x,|u|) u \cdot\left(z_{n}^{+}-z_{n}^{-}\right)=\lim _{n \rightarrow \infty} \operatorname{Re} \int_{\mathbb{R}^{3}} f_{\varepsilon}\left(x,\left|u_{n}\right|\right) u_{n} \cdot\left(z_{n}^{+}-z_{n}^{-}\right)=0 .
$$

Then subtracting the left and right sides of the two equations above, and using

$$
\begin{aligned}
& \lim _{n \rightarrow \infty} \operatorname{Re} \int_{\mathbb{R}^{3}} \tilde{\chi}(\varepsilon x) V_{\varepsilon}(x) \tilde{\xi}\left(x,\left|u_{n}\right|\right) u \cdot\left(z_{n}^{+}-z_{n}^{-}\right) \\
& =\lim _{n \rightarrow \infty} \operatorname{Re} \int_{\mathbb{R}^{3}} \tilde{\chi}(\varepsilon x) V_{\varepsilon}(x) \tilde{\xi}(x,|u|) u \cdot\left(z_{n}^{+}-z_{n}^{-}\right)=0,
\end{aligned}
$$


we obtain that

$$
\begin{aligned}
& \left\|z_{n}\right\|^{2}+\operatorname{Re} \int_{\mathbb{R}^{3}} V(\varepsilon x) z_{n} \cdot\left(z_{n}^{+}-z_{n}^{-}\right) \\
& \quad-\frac{1}{8} \tilde{\chi}(\varepsilon x) V(\varepsilon x) \tilde{\xi}\left(x,\left|u_{n}\right|\right) z_{n} \cdot\left(z_{n}^{+}-z_{n}^{-}\right)=o(1) .
\end{aligned}
$$

Similarly as the proof above, for $\Omega(n)=\left\{x \in \mathbb{R}^{3}:\left|u_{n}(x)\right| \geq 3 \phi(x)\right\}$, there holds

$$
\begin{aligned}
& \int_{\mathbb{R}^{3}} V(\varepsilon x)\left|z_{n}^{+}\right|^{2}-\frac{1}{8} \tilde{\chi}(\varepsilon x) V(\varepsilon x) \tilde{\xi}\left(x,\left|u_{n}\right|\right)\left|z_{n}^{+}\right|^{2} \\
& \geq \int_{\mathbb{R}^{3}}-\theta a(1-\tilde{\chi}(\varepsilon x))\left|z_{n}^{+}\right|^{2}-\frac{7 a}{8} \int_{\Omega(n)} \tilde{\chi}(\varepsilon x)\left|z_{n}^{+}\right|^{2}+\frac{1}{2} \int_{\mathbb{R}^{3}} \tilde{\chi}(\varepsilon x) V_{+}(\varepsilon x)\left|z_{n}^{+}\right|^{2} \\
& \quad+\int_{\mathbb{R}^{3} \backslash \Omega(n)} \tilde{\chi}(\varepsilon x) V_{-}(\varepsilon x)\left|z_{n}^{+}\right|^{2} .
\end{aligned}
$$

Since $z_{n} \rightarrow 0$ weakly in $E$, there holds $\left|z_{n}(x)\right| \rightarrow 0$ a.e. $x \in \mathbb{R}^{3}$. Combining this with $\left|\left(1-\chi_{n}\right) z_{n}\right| \leq\left|z_{n}\right|$, we know $\left|\left(1-\chi_{n}(x)\right) z_{n}(x)\right| \rightarrow 0$ a.e. $x \in \mathbb{R}^{3}$, where $\chi_{n}$ is the characteristic function of $\Omega(n)$. Moreover,

$\left|\left(1-\chi_{n}\right) z_{n}\right|^{2}=\left|\left(1-\chi_{n}\right)\left(u_{n}-u\right)\right|^{2} \leq 2\left|\left(1-\chi_{n}\right) u_{n}\right|^{2}+2|u|^{2} \leq 18 \phi^{2}+2|u|^{2} \in L^{1}\left(\mathbb{R}^{3}\right)$.

Then from Lebesgue dominated convergence theorem, it follows

$$
\lim _{n \rightarrow \infty} \int_{\mathbb{R}^{3} \backslash \Omega(n)}\left|z_{n}\right|^{2}=\lim _{n \rightarrow \infty} \int_{\mathbb{R}^{3}}\left|\left(1-\chi_{n}\right) z_{n}\right|^{2}=\int_{\mathbb{R}^{3}} \lim _{n \rightarrow \infty}\left|\left(1-\chi_{n}\right) z_{n}\right|^{2}=0,
$$

and hence

$$
\begin{aligned}
\int_{\mathbb{R}^{3} \backslash \Omega(n)} \tilde{\chi}(\varepsilon x) V_{-}(\varepsilon x)\left|z_{n}^{+}\right|^{2} & \geq \int_{\mathbb{R}^{3} \backslash \Omega(n)} \tilde{\chi}(\varepsilon x) V_{-}(\varepsilon x)\left(\left|z_{n}\right|^{2}+2\left|z_{n}\right|\left|z_{n}^{-}\right|+\left|z_{n}^{-}\right|^{2}\right) \\
& =\int_{\mathbb{R}^{3} \backslash \Omega(n)} \tilde{\chi}(\varepsilon x) V_{-}(\varepsilon x)\left|z_{n}^{-}\right|^{2}+o(1) .
\end{aligned}
$$

Therefore, we have

$$
\begin{aligned}
& \int_{\mathbb{R}^{3}} V(\varepsilon x)\left|z_{n}^{+}\right|^{2}-\frac{1}{8} \tilde{\chi}(\varepsilon x) V(\varepsilon x) \tilde{\xi}\left(x,\left|u_{n}\right|\right)\left|z_{n}^{+}\right|^{2} \\
& \quad \geq \int_{\mathbb{R}^{3}}-\theta a(1-\tilde{\chi}(\varepsilon x))\left|z_{n}^{+}\right|^{2}-\frac{7 a}{8} \int_{\Omega(n)} \tilde{\chi}(\varepsilon x)\left|z_{n}^{+}\right|^{2}+\frac{1}{2} \int_{\mathbb{R}^{3}} \tilde{\chi}(\varepsilon x) V_{+}(\varepsilon x)\left|z_{n}^{+}\right|^{2} \\
& \quad+\int_{\mathbb{R}^{3} \backslash \Omega(n)} \tilde{\chi}(\varepsilon x) V_{-}(\varepsilon x)\left|z_{n}^{-}\right|^{2}+o(1) .
\end{aligned}
$$

And similarly,

$$
\begin{aligned}
\int_{\mathbb{R}^{3}} & -V(\varepsilon x)\left|z_{n}^{-}\right|^{2}+\frac{1}{8} \tilde{\chi}(\varepsilon x) V(\varepsilon x) \tilde{\xi}\left(x,\left|u_{n}\right|\right)\left|z_{n}^{-}\right|^{2} \\
\geq & \int_{\mathbb{R}^{3}}-\theta a(1-\tilde{\chi}(\varepsilon x))\left|z_{n}^{-}\right|^{2}-\frac{7 a}{8} \int_{\Omega(n)} \tilde{\chi}(\varepsilon x)\left|z_{n}^{-}\right|^{2}-\frac{1}{2} \int_{\mathbb{R}^{3}} \tilde{\chi}(\varepsilon x) V_{-}(\varepsilon x)\left|z_{n}^{-}\right|^{2} \\
& -\int_{\mathbb{R}^{3} \backslash \Omega(n)} \tilde{\chi}(\varepsilon x) V_{+}(\varepsilon x)\left|z_{n}^{+}\right|^{2}+o(1) .
\end{aligned}
$$

Taking these two estimates into (2.14), we have $\min \left\{1-\theta, \frac{1}{8}\right\}\left\|z_{n}\right\|^{2} \leq o(1)$, which completes the proof. 


\section{Existence of solutions of the modified problem}

In this section, we shall use a linking theorem to obtain a nontrivial solution for the modified variational functional $\Phi_{\varepsilon}$. Before doing that, we first verify that the functional $\Phi_{\varepsilon}$ possesses the linking structure.

We define

$$
\begin{aligned}
& B_{r}=\{u \in E:\|u\| \leq r\}, \quad S_{r}=\{u \in E:\|u\|=r\} \\
& E(e)=\left\{u \in E: u=s e+v, s \geq 0 \text { and } v \in E^{-}\right\} .
\end{aligned}
$$

Lemma 3.1 There exists a positive constant $C$ (independent of $\varepsilon>0$ ), such that for any $u \in E$, there holds

$$
\left.\left|\int_{\mathbb{R}^{3}} V(\varepsilon x)\right| u\right|^{2}-2 Q_{\varepsilon}(u) \mid \leq \max \left\{\theta, \frac{7}{8}\right\}\|u\|^{2}+C \varepsilon^{5} .
$$

Proof By the definition of $\widehat{\xi}$, we know $\widehat{\xi}(x, t) \leq t^{2}$ for $t \geq 0$ and $\widehat{\xi}(x, t) \geq \frac{1}{2} t^{2}$ for $t \geq 6 \phi(x)$. So for $\Omega(u)=\left\{x \in \mathbb{R}^{3}:|u(x)| \geq 6 \phi(x)\right\}$, there holds

$$
\widehat{\xi}(x,|u(x)|) \geq \frac{1}{2}|u(x)|^{2}, x \in \Omega(u) .
$$

Consequently, we have

$$
\begin{aligned}
& \int_{\mathbb{R}^{3}} \tilde{\chi}(\varepsilon x) V(\varepsilon x)|u|^{2}-\frac{1}{4} \int_{\mathbb{R}^{3}} \tilde{\chi}(\varepsilon x) V(\varepsilon x) \widehat{\xi}(x,|u|) \\
& \leq \int_{\Omega(u)} \tilde{\chi}(\varepsilon x) V(\varepsilon x)|u|^{2}-\frac{1}{8} \tilde{\chi}(\varepsilon x) V_{+}(\varepsilon x)|u|^{2}-\frac{1}{4} \tilde{\chi}(\varepsilon x) V_{-}(\varepsilon x)|u|^{2} \\
& \quad+C \int_{\mathbb{R}^{3} \backslash \Omega(u)} \tilde{\chi}(\varepsilon x) \phi^{2} \\
& \quad \leq \frac{7 a}{8} \int_{\Omega(u)} \tilde{\chi}(\varepsilon x)|u|^{2}+C \varepsilon^{5}
\end{aligned}
$$

Then from (2.2) and (2.4), it follows that

$$
\begin{aligned}
\int_{\mathbb{R}^{3}} V(\varepsilon x)|u|^{2}-2 Q_{\varepsilon}(u)= & \int_{\mathbb{R}^{3}} \tilde{\chi}(\varepsilon x) V(\varepsilon x)|u|^{2}-\frac{1}{4} \int_{\mathbb{R}^{3}} \tilde{\chi}(\varepsilon x) V(\varepsilon x) \widehat{\xi}(x,|u|) \\
& +\int_{\mathbb{R}^{3}}(1-\tilde{\chi}(\varepsilon x)) V(\varepsilon x)|u|^{2} \\
\leq & \max \left\{\theta, \frac{7}{8}\right\}\|u\|^{2}+C \varepsilon^{5} .
\end{aligned}
$$

Similarly, one can prove

$$
\int_{\mathbb{R}^{3}} V(\varepsilon x)|u|^{2}-2 Q_{\varepsilon}(u) \geq-\max \left\{\theta, \frac{7}{8}\right\}\|u\|^{2}-C \varepsilon^{5},
$$

which completes the proof.

According to this lemma and $\left(f_{1}\right)$, for $u \in E^{+}$, there holds

$$
\begin{aligned}
\Phi_{\varepsilon}(u) & \geq \frac{1}{2}\|u\|^{2}-\frac{1}{2} \max \left\{\theta, \frac{7}{8}\right\}\|u\|^{2}-\frac{1}{p} \int_{\mathbb{R}^{3}}|u|^{p}-C \varepsilon^{5} \\
& \geq \frac{1}{2} \min \left\{1-\theta, \frac{1}{8}\right\}\|u\|^{2}-C\|u\|^{p}-C \varepsilon^{5} .
\end{aligned}
$$


This implies that there exist small $r_{0}>0$ and $\rho_{0}>0$, such that

$$
\inf _{u \in S_{r_{0}} \cap E^{+}} \Phi_{\varepsilon}(u) \geq \rho_{0}, \quad \inf _{u \in B_{r_{0}} \cap E^{+}} \Phi_{\varepsilon}(u) \geq-C \varepsilon^{5}, \quad \varepsilon>0 .
$$

Let $e_{0} \in E^{+} \backslash\{0\}$, then there exists $c_{0}>0$ such that

$$
\left|e_{0}\right|_{p} \geq c_{0}\left\|e_{0}\right\|
$$

For this $c_{0}$ and

$$
c_{\theta}:=\sqrt{\frac{\min \left\{1-\theta, \frac{1}{8}\right\}}{\min \left\{5-\theta, \frac{33}{8}\right\}},}
$$

we choose an $R_{0}>r_{0}$ satisfying that

$$
\frac{\min \left\{1-\theta, \frac{1}{8}\right\}}{4} R_{0}^{2} \geq 1 \text { and }\left(d_{p} c_{0} c_{\theta}\right)^{p} R_{0}^{p-2}-p \geq 1,
$$

where $d_{p}$ is the constant in Proposition 2.1.

Lemma 3.2 There exists $\varepsilon_{0}>0$, such that for $\varepsilon \in\left(0, \varepsilon_{0}\right),\left.\Phi_{\varepsilon}\right|_{E\left(e_{0}\right) \backslash B_{R_{0}}} \leq-1$. In particular, $\sup _{u \in E\left(e_{0}\right)} \Phi_{\varepsilon}(u) \leq 2 R_{0}^{2}$.

Proof Arguing indirectly, we assume that there exist sequence $\varepsilon_{j} \rightarrow 0$ as $j \rightarrow \infty$, and $u_{j} \in E\left(e_{0}\right) \backslash B_{R_{0}}$ such that $\Phi_{\varepsilon_{j}}\left(u_{j}\right) \geq-1$. Using Lemma 3.1, we have

$$
-1 \leq \frac{1}{2}\left\|u_{j}^{+}\right\|^{2}-\frac{1}{2}\left\|u_{j}^{-}\right\|^{2}+\frac{1}{2} \max \left\{\theta, \frac{7}{8}\right\}\left\|u_{j}\right\|_{2}^{2}+C \varepsilon_{j}^{5}-F_{\varepsilon_{j}}\left(x,\left|u_{j}\right|\right) .
$$

This, together with $\left\|u_{j}\right\| \geq R_{0}$ and the former inequality in (3.3), yields that

$$
0 \leq\left\|u_{j}^{+}\right\|^{2}-\frac{\min \left\{1-\theta, \frac{1}{8}\right\}}{4}\left\|u_{j}^{-}\right\|^{2}+C \varepsilon_{j}^{5}-F_{\varepsilon_{j}}\left(x,\left|u_{j}\right|\right) .
$$

Therefore,

$$
\left\|u_{j}^{-}\right\|^{2} \leq \frac{4}{\min \left\{5-\theta, \frac{33}{8}\right\}}\left\|u_{j}\right\|^{2}+o_{j}(1),\left\|u_{j}^{+}\right\|^{2} \geq \frac{\min \left\{1-\theta, \frac{1}{8}\right\}}{\min \left\{5-\theta, \frac{33}{8}\right\}}\left\|u_{j}\right\|^{2}+o_{j}(1) .
$$

Since $u_{j} \in E\left(e_{0}\right)$, if $\frac{u_{j}}{\left\|u_{j}\right\|} \rightarrow v$ weakly in $E$, then $\frac{u_{j}^{+}}{\left\|u_{j}\right\|} \rightarrow v^{+}$with $v^{+}=t_{0} e_{0}$ and

$$
\left\|v^{+}\right\|^{2} \geq \frac{\min \left\{1-\theta, \frac{1}{8}\right\}}{\min \left\{5-\theta, \frac{33}{8}\right\}}=c_{\theta}^{2} .
$$

Taking the fact $F_{\varepsilon}(x, t) \geq \frac{1}{p}(1-\chi(\varepsilon x)) t^{p}$ into (3.4),

$$
0 \leq\left\|u_{j}^{+}\right\|^{2}+C \varepsilon_{j}^{5}-\frac{1}{p} \int_{\mathbb{R}^{3}}\left(1-\chi\left(\varepsilon_{j} x\right)\right)\left|u_{j}\right|^{p},
$$

and multiplying both sides of this inequality by $\frac{1}{\left\|u_{j}\right\|^{p}}$, we deduce that

$$
\frac{1}{p} \int_{\mathbb{R}^{3}}\left(1-\chi\left(\varepsilon_{j} x\right)\right)\left|\frac{u_{j}}{\left\|u_{j}\right\|}\right|^{p} \leq \frac{1}{\left\|u_{j}\right\|^{p-2}}+o_{j}(1) .
$$


Since as $j \rightarrow \infty,\left(1-\chi\left(\varepsilon_{j} x\right)\right)\left|\frac{u_{j}(x)}{\left\|u_{j}\right\|}\right|^{p} \rightarrow|v(x)|^{p}$ a.e. $x \in \mathbb{R}^{3}$, it follows from Fatou's lemma and $\left\|u_{j}\right\| \geq R_{0}$ that

$$
\frac{1}{p} \int_{\mathbb{R}^{3}}|v|^{p} \leq \frac{1}{p} \liminf _{j \rightarrow \infty} \int_{\mathbb{R}^{3}}\left(1-\chi\left(\varepsilon_{j} x\right)\right)\left|\frac{u_{j}}{\left\|u_{j}\right\|}\right|^{p} \leq \frac{1}{R_{0}^{p-2}} .
$$

Combining this with Proposition 2.1, (3.2), (3.5) and $v^{+}=t_{0} e_{0}$, we have

$$
d_{p}^{p} c_{0}^{p} c_{\theta}^{p} \leq d_{p}^{p} c_{0}^{p}\left\|v^{+}\right\|^{p} \leq d_{p}^{p} \int_{\mathbb{R}^{3}}\left|v^{+}\right|^{p} \leq \int_{\mathbb{R}^{3}}|v|^{p} \leq \frac{p}{R_{0}^{p-2}},
$$

which is a contradiction to (3.3).

To show $\sup _{u \in E\left(e_{0}\right)} \Phi_{\varepsilon}(u) \leq 2 R_{0}^{2}$, we only need to show

$$
\sup _{u \in E\left(e_{0}\right) \cap B_{R_{0}}} \Phi_{\varepsilon}(u) \leq 2 R_{0}^{2} .
$$

In fact, for any $u \in B_{R_{0}}$, there holds

$$
\Phi_{\varepsilon}(u) \leq \frac{1}{2}\left\|u^{+}\right\|^{2}-\frac{1}{2}\left\|u^{-}\right\|^{2}+\frac{3 a}{4}|u|_{2}^{2} \leq 2 R_{0}^{2} .
$$

Let $\mathcal{S}$ be a countable dense subset of $\left(E^{-}\right)^{*}$ (the dual space of $E^{-}$) and $D=\left\{d_{s}: s \in\right.$ $\mathcal{S}, d_{s}(u, v)=|s(u-v)|$ for $\left.u, v \in E^{-}\right\}$be the associated family of semimetrics on $E^{-}$. Let $\mathcal{P}$ be the family of seminorms on $E$ consisting of all seminorms:

$$
p_{s}: E \rightarrow \mathbb{R} ; \quad p_{s}(u)=\left|s\left(u^{-}\right)\right|+\left\|u^{+}\right\|, u \in E, s \in \mathcal{S} .
$$

Thus $\mathcal{P}$ is countable and it induces the product topology on $E$ given by the $D$-topology on $E^{-}$and the norm topology on $E^{+}$. We denote this topology by $\left(E, \mathcal{I}_{\mathcal{P}}\right)$ and denote the weak* topology on $E^{*}$ by $\left(E^{*}, \mathcal{T}_{w^{*}}\right)$. Then we can prove that:

Lemma 3.3 For any $\varepsilon>0, b \in \mathbb{R}$ and $\Phi_{\varepsilon, b}=\left\{u \in E: \Phi_{\varepsilon}(u) \geq b\right\}$, there holds $\Phi_{\varepsilon, b}$ is $\mathcal{T}_{\mathcal{P}}$-closed and holds $\Phi_{\varepsilon}^{\prime}:\left(\Phi_{\varepsilon, b}, \mathcal{T}_{\mathcal{P}}\right) \rightarrow\left(E^{*}, \mathcal{T}_{w^{*}}\right)$ is continuous.

Proof Let $\left\{u_{n}\right\} \subset \Phi_{\varepsilon, b}$ be a sequence $\mathcal{T}_{\mathcal{P}}$-converges to $u$ in $E$. Then $u_{n}^{+} \rightarrow u^{+}$strongly in $E$ and hence $u_{n}^{+}$is bounded in $E$. Similarly as that in (3.4), by Lemma 3.1, it is easy to see

$$
b \leq \Phi_{\varepsilon}\left(u_{n}\right) \leq\left\|u_{n}^{+}\right\|^{2}-\frac{\min \left\{1-\theta, \frac{1}{8}\right\}}{2}\left\|u_{n}^{-}\right\|^{2}+C \varepsilon^{5},
$$

which implies the boundedness of $u_{n}^{-}$in $E$. Thus $u_{n} \rightarrow u$ and $u_{n}^{-} \rightarrow u^{-}$weakly in $E$. To show $\Phi_{\varepsilon}(u) \geq b$, we write

$$
\begin{aligned}
\Phi_{\varepsilon}\left(u_{n}\right)= & \frac{1}{2}\left\|u_{n}^{+}\right\|^{2}+\frac{1}{2} \int_{\mathbb{R}^{3}} V(\varepsilon x)\left(\left|u_{n}^{+}\right|^{2}+2 \operatorname{Re} u_{n}^{+} \cdot u_{n}^{-}\right)-\int_{\mathbb{R}^{3}} F_{\varepsilon}\left(x,\left|u_{n}\right|\right) \\
& -\frac{1}{2}\left\|u_{n}^{-}\right\|^{2}+\frac{1}{2} \int_{\mathbb{R}^{3}} V(\varepsilon x)\left|u_{n}^{-}\right|^{2}-\frac{1}{8} \int_{\mathbb{R}^{3}} \tilde{\chi}(\varepsilon x)\left(V_{+}(\varepsilon x)+V_{-}(\varepsilon x)\right) \hat{\xi}\left(x,\left|u_{n}\right|\right) .
\end{aligned}
$$

Note that $u_{n}^{+} \rightarrow u^{+}$strongly in $E, \int_{\mathbb{R}^{3}} F_{\varepsilon}(x,|\cdot|)$ is weakly continuous in $E$ and that $\int_{\mathbb{R}^{3}} \tilde{\chi}(\varepsilon x) V_{+}(\varepsilon x) \hat{\xi}(x,|\cdot|)$ is weakly lower semicontinuous in $E$. We only need to show

$$
\left\|u^{-}\right\|^{2}-\int_{\mathbb{R}^{3}} V(\varepsilon x)\left|u^{-}\right|^{2}+2 Q_{\varepsilon}^{-}(u) \leq \liminf _{n \rightarrow \infty}\left(\left\|u_{n}^{-}\right\|^{2}-\int_{\mathbb{R}^{3}} V(\varepsilon x)\left|u_{n}^{-}\right|^{2}+2 Q_{\varepsilon}^{-}\left(u_{n}\right)\right),
$$


where

$$
Q_{\varepsilon}^{-}(u)=\frac{1}{8} \int_{\mathbb{R}^{3}} \tilde{\chi}(\varepsilon x) V_{-}(\varepsilon x) \widehat{\xi}(x,|u|), \quad\left(Q_{\varepsilon}^{-}\right)^{\prime}(u) v=\frac{1}{8} \int_{\mathbb{R}^{3}} \tilde{\chi}(\varepsilon x) V_{-}(\varepsilon x) \tilde{\xi}(x,|u|) u \cdot v .
$$

In fact, since $u_{n} \rightarrow u$ in $E^{+}, u_{n} \rightarrow u$ weakly in $E$ and $\widetilde{\xi} \in[0,2]$, there holds

$$
\begin{aligned}
\left|Q_{\varepsilon}^{-}\left(u_{n}\right)-Q_{\varepsilon}^{-}(u)\right| & \leq \sup _{s \in[0,1]}\left|\left(Q_{\varepsilon}^{-}\right)^{\prime}\left(u+s\left(u_{n}-u\right)\right)\left(u_{n}-u\right)\right| \\
& \leq \frac{1}{4} \sup _{s \in[0,1]} \int_{\mathbb{R}^{3}} \tilde{\chi}(\varepsilon x)\left|V_{-}(\varepsilon x)\right|\left|u+s\left(u_{n}-u\right)\right|\left|u_{n}-u\right| \\
& \leq \frac{1}{4} \int_{\mathbb{R}^{3}} \tilde{\chi}(\varepsilon x)\left|V_{-}(\varepsilon x)\right|\left|u_{n}^{-}-u^{-}\right|^{2}+o(1) .
\end{aligned}
$$

Consequently, we have

$$
\begin{aligned}
& \left\|u_{n}^{-}\right\|^{2}-\left\|u^{-}\right\|^{2}-\int_{\mathbb{R}^{3}} V(\varepsilon x)\left(\left|u_{n}^{-}\right|^{2}-\left|u^{-}\right|^{2}\right)+2\left(Q_{\varepsilon}^{-}\left(u_{n}\right)-Q_{\varepsilon}^{-}(u)\right) \\
& \geq\left\|u_{n}^{-}-u^{-}\right\|^{2}-\int_{\mathbb{R}^{3}} V(\varepsilon x)\left|u_{n}^{-}-u^{-}\right|^{2}-\frac{1}{2} \int_{\mathbb{R}^{3}} \tilde{\chi}(\varepsilon x)\left|V_{-}(\varepsilon x)\right|\left|u_{n}^{-}-u^{-}\right|^{2}+o(1) \\
& \geq\left\|u_{n}^{-}-u^{-}\right\|^{2}-\int_{\mathbb{R}^{3}}(1-\tilde{\chi}(\varepsilon x)) V(\varepsilon x)\left|u_{n}^{-}-u^{-}\right|^{2}+\tilde{\chi}(\varepsilon x) V_{+}(\varepsilon x)\left|u_{n}^{-}-u^{-}\right|^{2} \\
& \quad-\int_{\mathbb{R}^{3}} \tilde{\chi}(\varepsilon x) V_{-}(\varepsilon x)\left|u_{n}^{-}-u^{-}\right|^{2}-\frac{1}{2} \int_{\mathbb{R}^{3}} \tilde{\chi}(\varepsilon x)\left|V_{-}(\varepsilon x) \| u_{n}^{-}-u^{-}\right|^{2}+o(1) \\
& \geq\left\|u_{n}^{-}-u^{-}\right\|^{2}-a \int_{\mathbb{R}^{3}}\left|u_{n}^{-}-u^{-}\right|^{2}+o(1) \geq o(1) .
\end{aligned}
$$

Thus $\Phi_{\varepsilon, b}$ is $\mathcal{T}_{\mathcal{P}}$-closed for any $b \in \mathbb{R}$.

To show $\Phi_{\varepsilon}^{\prime}:\left(\Phi_{\varepsilon, b}, \mathcal{T}_{\mathcal{P}}\right) \rightarrow\left(E^{*}, \mathcal{T}_{w^{*}}\right)$ is continuous, we suppose $\left\{u_{n}\right\} \mathcal{T}_{\mathcal{P}}$-converges toward $u$ in $\Phi_{\varepsilon, b}$. By the same reason as above, $\left\{u_{n}\right\}$ is bounded and converges weakly to $u$ in $E$. And then from (2.9), the conclusion follows.

Then, for the fixed $e_{0} \in E^{+}$, we set

$$
\mathcal{Q}\left(e_{0}\right)=\left\{u \in E\left(e_{0}\right): u=s e_{0}+v, s>0, v \in E^{-} \text {and }\|u\|<R_{0}\right\},
$$

with $R_{0}$ the positive constant we defined in (3.3). Obviously, by Lemma 3.1, for any $v \in E^{-}$ and $\varepsilon>0$, there holds

$$
\Phi_{\varepsilon}(v) \leq-\frac{1}{2}\|v\|^{2}+\frac{1}{2} \max \left\{\theta, \frac{7}{8}\right\}\|v\|^{2}+C \varepsilon^{5}-\int_{\mathbb{R}^{3}} F_{\varepsilon}(x,|v|) \leq C \varepsilon^{5} .
$$

Combining this with Lemma 3.2 and the former inequality in (3.1), if we make $\varepsilon_{0}$ smaller if necessary, we shall have

$$
\sup _{u \in \partial \mathcal{Q}\left(e_{0}\right)} \Phi_{\varepsilon}(u)<\inf _{u \in S_{r_{0}} \cap E^{+}} \Phi_{\varepsilon}(u), \quad \varepsilon \in\left(0, \varepsilon_{0}\right) .
$$

Thus, according to Lemma 3.3, we may apply a linking theorem (see Theorem 4.4 in [14]) and obtain a Palais-Smale sequence at level

$$
c_{\varepsilon} \in\left[\inf _{u \in S_{r_{0}} \cap E^{+}} \Phi_{\varepsilon}(u), \sup _{u \in \mathcal{Q}\left(e_{0}\right)} \Phi_{\varepsilon}(u)\right] \subset\left[\rho_{0}, 2 R_{0}^{2}\right], \varepsilon \in\left(0, \varepsilon_{0}\right) .
$$

Then by Lemma 2.3 and (2.13), we have the following theorem. 
Theorem 3.4 For every $\varepsilon \in\left(0, \varepsilon_{0}\right)$, there exists a nontrivial solution $w_{\varepsilon}$ for the modified variational functional $\Phi_{\varepsilon}$. Moreover, there holds

$$
\left\|w_{\varepsilon}\right\| \leq C_{R_{0}}
$$

where $C_{R_{0}}$ depends only on $R_{0}$, but is independent of $\varepsilon$.

\section{Localization of solutions and the Proof of Theorem 1.1}

In this section, we shall show that the critical point $w_{\varepsilon}$ for the modified problem given in Theorem 3.4 is actually a solution of original problem $\left(D_{\varepsilon}\right)$ when $\varepsilon$ is sufficiently small. We need several lemmas first for some necessary estimates.

Lemma 4.1 For $\varepsilon \in\left(0, \varepsilon_{0}\right)$, let $w_{\varepsilon}$ be the solution of $\Phi_{\varepsilon}$ which is given in Theorem 3.4. Then for any $q \geq 2, w_{\varepsilon} \in W^{1, q}\left(\mathbb{R}^{3}, \mathbb{C}^{4}\right)$ and $\left\|w_{\varepsilon}\right\|_{W^{1, q}} \leq C$, where $C$ depends only on $q$. Moreover, $w_{\varepsilon} \in W_{\text {loc }}^{2, q}\left(\mathbb{R}^{3}, \mathbb{C}^{4}\right)$ for $q \in[1,+\infty)$ and there exists a constant independent of $\varepsilon \in\left(0, \varepsilon_{0}\right)$ such that for any $x \in \mathbb{R}^{3}$

$$
\left|w_{\varepsilon}(x)\right| \leq C_{0}\left|w_{\varepsilon}\right|_{2, B(x, 1)} .
$$

Proof Let $D=-i \alpha \cdot \nabla$. Then $w_{\varepsilon}$ satisfies

$$
D w_{\varepsilon}+a \beta w_{\varepsilon}=-V(\varepsilon x) w_{\varepsilon}+\frac{1}{8} \tilde{\chi}(\varepsilon x) V(\varepsilon x) \tilde{\xi}\left(x,\left|w_{\varepsilon}\right|\right) w_{\varepsilon}+f_{\varepsilon}\left(x,\left|w_{\varepsilon}\right|\right) w_{\varepsilon} .
$$

Since $\tilde{\chi} \in[0,1], \widetilde{\xi} \in[0,2]$ and $\left\|w_{\varepsilon}\right\| \leq C_{R_{0}}$, we can use the same iterative argument as that in [22] Proposition 3.2 and [20] Lemma 3.19, to obtain that $w_{\varepsilon} \in W^{1, q}\left(\mathbb{R}^{3}, \mathbb{C}^{4}\right)$ with $\left\|w_{\varepsilon}\right\|_{W^{1, q}} \leq C_{q}$, where $C_{q}$ depends only on $q$ for any $q \geq 2$. In particular, $w_{\varepsilon}$ is uniformly bounded in $L^{\infty}\left(\mathbb{R}^{3}, \mathbb{C}^{4}\right)$ for $\varepsilon \in\left(0, \varepsilon_{0}\right)$.

Setting

$$
h_{\varepsilon}\left(x,\left|w_{\varepsilon}\right|\right):=-V(\varepsilon x)+\frac{1}{8} \widetilde{\chi}(\varepsilon x) V(\varepsilon x) \tilde{\xi}\left(x,\left|w_{\varepsilon}\right|\right)+f_{\varepsilon}\left(x,\left|w_{\varepsilon}\right|\right),
$$

we claim $h_{\varepsilon}\left(x,\left|w_{\varepsilon}\right|\right) w_{\varepsilon} \in W_{\text {loc }}^{1, q}$ for any $q \geq 1$. In fact, for any fixed $q_{0} \geq 2, y \in \mathbb{R}^{3}$ and $r>0, D^{h} w_{\varepsilon}$, the difference quotient of $w_{\varepsilon}$, is uniformly bounded in $L^{q_{0}}(B(y, r))$ for $h \in(0,1)$. Combining this with the fact that $w_{\varepsilon} \in W^{1, q}\left(\mathbb{R}^{3}\right), V$ and $\tilde{\chi}$ are of class $C^{1}$ and the definitions of $\widetilde{\xi}$ and $f_{\varepsilon}$, we can deduce that $D^{h}\left(h_{\varepsilon}\left(x,\left|w_{\varepsilon}\right|\right) w_{\varepsilon}\right)$ is uniformly bounded in $L^{q_{0}}(B(y, r))$ for $h \in(0,1)$. Hence $h_{\varepsilon}\left(x,\left|w_{\varepsilon}\right|\right) w_{\varepsilon} \in W^{1, q_{0}}(B(y, r))$ and the claim is true.

Thus we may apply the operator $D$ on the two sides of (4.2), since $D^{2}=-\Delta$,

$$
\Delta w_{\varepsilon}=D\left(a \beta w_{\varepsilon}-h_{\varepsilon}\left(x,|w|_{\varepsilon}\right) w_{\varepsilon}\right) .
$$

Note that, for $q \geq 1, h_{\varepsilon}\left(x,\left|w_{\varepsilon}\right|\right) w_{\varepsilon} \in W_{\text {loc }}^{1, q}$. So the right-hand side of this equality belongs to $L_{\text {loc }}^{q}$, and hence we have $w_{\varepsilon} \in W_{\text {loc }}^{2, q}\left(\mathbb{R}^{3}, \mathbb{C}^{4}\right), q \in[1,+\infty)$. Then in (4.3), using (4.2) and the definition of $D$, we have that, for $x \in \mathbb{R}^{3}$ such that $w_{\varepsilon}(x) \neq 0$, there holds

$$
\Delta w_{\varepsilon}=a^{2} w_{\varepsilon}-\left(h_{\varepsilon}\left(x,\left|w_{\varepsilon}\right|\right)\right)^{2} w_{\varepsilon}-i \sum_{k=1}^{3} \partial_{k}\left(h_{\varepsilon}\left(x,\left|w_{\varepsilon}\right|\right)\right) \alpha_{k} w_{\varepsilon}
$$

and that, for $x \in \mathbb{R}^{3}$ such that $w_{\varepsilon}(x)=0$, there holds

$$
\Delta w_{\varepsilon}=0
$$


Setting

$$
\operatorname{sgn} u= \begin{cases}u /|u|, & u \neq 0 \\ 0, & u=0\end{cases}
$$

by Kato's inequality [11],

$$
\Delta\left|w_{\varepsilon}\right| \geq \operatorname{Re}\left[\Delta w_{\varepsilon} \cdot\left(\operatorname{sgn} w_{\varepsilon}\right)\right]
$$

and the fact

$$
\operatorname{Re}\left[-i \sum_{k=1}^{3} \partial_{k}\left(h_{\varepsilon}\left(x,\left|w_{\varepsilon}\right|\right)\right) \alpha_{k} w_{\varepsilon} \cdot \frac{w_{\varepsilon}}{\left|w_{\varepsilon}\right|}\right]=0
$$

we deduce

$$
\Delta\left|w_{\varepsilon}\right| \geq a^{2}\left|w_{\varepsilon}\right|-\left(h_{\varepsilon}\left(x,\left|w_{\varepsilon}\right|\right)\right)^{2}\left|w_{\varepsilon}\right| .
$$

Since $w_{\varepsilon}$ is uniformly bounded in $L^{\infty}\left(\mathbb{R}^{3}, \mathbb{C}^{4}\right)$, there exists a constant $M>0$ such that

$$
\Delta\left|w_{\varepsilon}\right| \geq-M\left|w_{\varepsilon}\right|
$$

Then it follows from the sub-solution estimate [26] that

$$
\left|w_{\varepsilon}(x)\right| \leq C_{0}\left|w_{\varepsilon}\right|_{2, B(x, 1)}
$$

uniformly holds for $\varepsilon \in\left(0, \varepsilon_{0}\right)$ with some positive constant $C_{0}$.

Lemma 4.2 For any sequence $\varepsilon \rightarrow 0$, up to a subsequence if necessary, there exist $m \in \mathbb{N}$, $m$ nonzero functions $w_{j}$ in $E, 1 \leq j \leq m$ and $m$ sequences $\left\{y_{\varepsilon, j}\right\}, 1 \leq j \leq m$ such that,

(i) for $1 \leq j \neq j^{\prime} \leq m$,

$$
\left|y_{\varepsilon, j}\right| \rightarrow \infty, \quad\left|y_{\varepsilon, j}-y_{\varepsilon, j^{\prime}}\right| \rightarrow \infty, \quad \varepsilon y_{\varepsilon, j} \rightarrow y_{j} \in \Lambda^{\delta_{1}}
$$

(ii) there holds

$$
w_{\varepsilon, m}:=w_{\varepsilon}-w_{0}-\sum_{j=1}^{m} w_{j}\left(\cdot-y_{\varepsilon, j}\right) \rightarrow 0 \text { in } L^{q}\left(\mathbb{R}^{3}, \mathbb{C}^{4}\right), q \in(2,3),
$$

where $w_{0} \in E$ is a solution of

$$
-i \alpha \cdot \nabla u+a \beta u+V(0) u=|u|^{p-2} u
$$

and $w_{j} \in E$ is a nontrivial solution of

$$
-i \alpha \cdot \nabla u+a \beta u+V\left(y_{j}\right) u=\left(1-\chi\left(y_{j}\right)\right)|u|^{p-2} u, \quad 1 \leq j \leq m .
$$

Moreover, when $m=0$, we believe that $w_{\varepsilon} \rightarrow w_{0}$ in $L^{q}\left(\mathbb{R}^{3}, \mathbb{C}^{4}\right), q \in(2,3)$.

Proof Since the results of this lemma are quite standard, we give the details of proof in "Appendix." 
Next, for any $\varepsilon>0$, let

$$
y_{\varepsilon, 0}=0 .
$$

Let $\varepsilon_{n}$ be a sequence such that

$$
\lim _{n \rightarrow \infty} \varepsilon_{n}=0 \text {. }
$$

For sequence $\left\{w_{\varepsilon_{n}}\right\}$, we apply Lemma 4.2 and obtain a subsequence (still denote by itself) such that statements (i), (ii) of Lemma 4.2 hold. In particular, for every integer $j \in[0, m]$, $\lim _{\varepsilon \rightarrow 0} \varepsilon_{n} y_{\varepsilon_{n}, j}$ exists. We may write the set of these limiting points by

$$
\left\{x_{0}, x_{1}, \ldots, x_{s}\right\}=\left\{\lim _{n \rightarrow \infty} \varepsilon_{n} y_{\varepsilon_{n}, j}: 0 \leq j \leq m\right\},
$$

such that $0 \leq s \leq m$ and $x_{j} \neq x_{j^{\prime}}$ for $0 \leq j \neq j^{\prime} \leq s$. Define

$$
\vartheta= \begin{cases}\frac{1}{10} \min _{0 \leq j \neq j^{\prime} \leq s}\left\{\left|x_{j}-x_{j^{\prime}}\right|, 1\right\}, & s \geq 1 ; \\ \frac{1}{10}, & s=0 .\end{cases}
$$

Proposition 4.3 Assume $\delta \in(0, \vartheta)$. Then there exist $C>0$ and $c>0$ independent of $n$ such that, for every $0 \leq j \leq m$, when $n$ is large enough,

$$
\left|w_{\varepsilon_{n}}(x)\right| \leq C \exp \left(-c \varepsilon_{n}^{-1}\right), \quad x \in \overline{B\left(y_{\varepsilon_{n}, j}, \delta \varepsilon_{n}^{-1}+1\right)} \backslash B\left(y_{\varepsilon_{n}, j}, \delta \varepsilon_{n}^{-1}-1\right) .
$$

Proof We define

$$
A_{n, j}=\overline{B\left(y_{\varepsilon_{n}, j}, \frac{3}{2} \delta \varepsilon_{n}^{-1}\right)} \backslash B\left(y_{\varepsilon_{n}, j}, \frac{1}{2} \delta \varepsilon_{n}^{-1}\right) .
$$

Then the definition of $\vartheta$ and the fact $0<\delta<\vartheta$ lead us to

$$
\operatorname{dist}\left(y_{\varepsilon_{n}, j^{\prime}}, A_{n, j}\right) \rightarrow \infty \text {, as } n \rightarrow \infty \text { for } 0 \leq j^{\prime}, j \leq m .
$$

Using (ii) of Lemma 4.2, (4.6) and

$$
\lim _{R \rightarrow \infty} \int_{\mathbb{R}^{3} \backslash B\left(y_{\varepsilon_{n}, j}, R\right)}\left|w_{j}\left(\cdot-y_{\varepsilon_{n}, j}\right)\right|^{p}=0, \quad 0 \leq j \leq m,
$$

we deduce that, for every $0 \leq j \leq m$,

$$
\lim _{n \rightarrow \infty} \sup _{z \in A_{n, j}} \int_{B(z, 1)}\left|w_{\varepsilon_{n}}\right|^{p}=0 .
$$

This, together with (4.1), yields that

$$
\left|w_{\varepsilon_{n}}(x)\right|=o_{n}(1), x \in A_{n, j}, 0 \leq j \leq m .
$$

For nonnegative integer $l$, we set

$$
D_{l}=\overline{B\left(y_{\varepsilon_{n}, j}, \frac{3}{2} \delta \varepsilon_{n}^{-1}-l\right)} \backslash B\left(y_{\varepsilon_{n}, j}, \frac{1}{2} \delta \varepsilon_{n}^{-1}+l\right)
$$

and set $\eta_{l}$ be a cutoff function satisfying that $0 \leq \eta_{l}(t) \leq 1,\left|\eta_{l}^{\prime}(t)\right| \leq 4$ for all $t$ and

$$
\eta_{l}(t)= \begin{cases}0, & t \leq \frac{1}{2} \delta \varepsilon_{n}^{-1}+l-1 \text { or } t \geq \frac{3}{2} \delta \varepsilon_{n}^{-1}-l+1 \\ 1, & \frac{1}{2} \delta \varepsilon_{n}^{-1}+l \leq t \leq \frac{3}{2} \delta \varepsilon_{n}^{-1}-l\end{cases}
$$


For $x \in \mathbb{R}^{3}$, let $\psi_{l}(x)=\eta_{l}\left(\left|x-y_{\varepsilon_{n}, j}\right|\right)$. Noting that $\varepsilon_{n} y_{\varepsilon_{n}, j} \rightarrow y_{j} \in \Lambda^{\delta_{1}}$, then by $\Lambda^{\delta_{1}+1} \subset$ $B(0, \widetilde{R} / 2),(4.5)$ and the choice of $\delta$, we have for $n \geq n_{0}$,

$$
\varepsilon_{n} x \in B(0, \widetilde{R}) \text { for any } x \in A_{n, j} .
$$

So for $l \geq 1$ and $x \in D_{l-1} \subset A_{n, j}$, there holds $\tilde{\chi}\left(\varepsilon_{n} x\right)=0$. Then multiplying both sides of (4.4):

$$
\Delta\left|w_{\varepsilon_{n}}\right| \geq a^{2}\left|w_{\varepsilon_{n}}\right|-\left(h_{\varepsilon_{n}}\left(x,\left|w_{\varepsilon_{n}}\right|\right)\right)^{2}\left|w_{\varepsilon_{n}}\right|
$$

by $\psi_{l}^{2}\left|w_{\varepsilon_{n}}\right|$ and integrating on $\mathbb{R}^{3}$, we have

$$
\begin{gathered}
\int_{D_{l-1}}|\nabla| w_{\varepsilon_{n}}||^{2} \psi_{l}^{2}+\left(a^{2}-\left(V\left(\varepsilon_{n} x\right)-f_{\varepsilon_{n}}\left(x,\left|w_{\varepsilon_{n}}\right|\right)\right)^{2}\right)\left|w_{\varepsilon_{n}}\right|^{2} \psi_{l}^{2} \\
\quad \leq-2 \int_{D_{l-1}} \psi_{l}\left|w_{\varepsilon_{n}}\right| \nabla \psi_{l} \nabla\left|w_{\varepsilon_{n}}\right| \leq 8 \int_{D_{l-1} \backslash D_{l}}\left|w_{\varepsilon_{n}}\right||\nabla| w_{\varepsilon_{n}}|| .
\end{gathered}
$$

By (2.4) and (4.8), for $l \geq 1$ and $x \in D_{l-1} \subset A_{n, j}$, there holds $\left|V\left(\varepsilon_{n} x\right)\right| \leq \theta a$, combining which with $\left(f_{1}\right)$ and (4.7), we have, for $n \geq n_{0}$

$$
\left|V\left(\varepsilon_{n} x\right)-f_{\varepsilon_{n}}\left(x,\left|w_{\varepsilon_{n}}\right|\right)\right| \leq(1+\theta) a / 2, \quad x \in D_{l-1} .
$$

Taking this into (4.9), we can deduce that

$$
\int_{D_{l}}|\nabla| w_{\varepsilon_{n}}||^{2}+\theta^{\prime}\left|w_{\varepsilon_{n}}\right|^{2} \leq 8 \int_{D_{l-1} \backslash D_{l}}\left|w_{\varepsilon_{n}}\right||\nabla| w_{\varepsilon_{n}}||, \quad \theta^{\prime}>0 .
$$

Setting $a_{l}=\int_{D_{l}}|\nabla| w_{\varepsilon_{n}}||^{2}+\left|w_{\varepsilon_{n}}\right|^{2}$, then by (4.1) and the same iteration arguments as that in [33] Proposition 4.4 (see also [10]), we obtain the conclusion.

Lemma 4.4 For any $1 \leq j \leq m, \lim _{\varepsilon \rightarrow 0} \operatorname{dist}\left(\varepsilon y_{\varepsilon, j}, \mathcal{V}\right)=0$.

Proof Arguing indirectly, we assume that there exist $1 \leq j \leq m$ and sequence $\left\{\varepsilon_{n}\right\}$ such that $\lim _{n \rightarrow \infty} \varepsilon_{n}=0$ and

$$
\operatorname{dist}\left(\varepsilon_{n} y_{\varepsilon_{n}, j}, \mathcal{V}\right)>0, \quad n \in \mathbb{N} .
$$

In (2.3), we have $\delta_{1}>0$ such that for any $y \in \Lambda^{\delta_{1}}$, if $B\left(y, \delta_{1}\right) \backslash \bar{\Lambda} \neq \emptyset$, there holds

$$
\inf _{x \in B\left(y, \delta_{1}\right) \backslash \bar{\Lambda}} \nabla V(y) * \nabla \operatorname{dist}(x, \Lambda)>0 .
$$

Since $\lim _{n \rightarrow \infty} \varepsilon_{n} y_{\varepsilon_{n}, j}=y_{j} \notin \mathcal{V}$, we know that there exists $\delta_{2}>0$ such that, for sufficiently large $n$,

$$
\inf _{x \in B\left(y_{\varepsilon_{n}, j}, \delta_{2} \varepsilon_{n}^{-1}\right)} \nabla V\left(\varepsilon_{n} x\right) * \nabla V\left(\varepsilon_{n} y_{\varepsilon_{n}, j}\right) \geq \frac{1}{2}\left|\nabla V\left(y_{j}\right)\right|^{2}>0 .
$$

Let $\vartheta$ be the positive constant we defined in (4.5), and we choose a $\delta_{0}$ satisfying

$$
0<\delta_{0}<\min \left\{\delta_{1}, \delta_{2}, \vartheta\right\}
$$

For simplicity, we denote by

$$
w_{n}=w_{\varepsilon_{n}}
$$


Then according to the definition of $\delta_{0}$ and Proposition 4.3, there exist $C>0$ and $c>0$ independent of $n$ such that, when $n$ is large enough,

$$
\left.\left|w_{n}(x)\right| \leq C \exp \left(-c \varepsilon_{n}^{-1}\right), \quad x \in \overline{B\left(y_{\varepsilon_{n}}, j\right.}, \delta_{0} \varepsilon_{n}^{-1}+1\right) \backslash B\left(y_{\varepsilon_{n}, j}, \delta_{0} \varepsilon_{n}^{-1}-1\right) .
$$

By Lemma 4.1, there exists $C>0$ independent of $n$ such that,

$$
\int_{\delta_{0} \varepsilon_{n}^{-1}-1}^{\delta_{0} \varepsilon_{n}^{-1}} \int_{\partial B\left(y_{\varepsilon_{n}, j}, r\right)}\left|\nabla w_{n}\right|^{2} \mathrm{~d} s \mathrm{~d} r \leq \int_{\mathbb{R}^{3}}\left|\nabla w_{n}\right|^{2} \leq C,
$$

which implies the existence of $\delta_{n}$ satisfying $-1 \leq \delta_{n} \varepsilon_{n}^{-1}-\delta_{0} \varepsilon_{n}^{-1} \leq 0$ and

$$
\int_{\partial B\left(y_{\varepsilon_{n}, j}, \delta_{n} \varepsilon_{n}^{-1}\right)}\left|\nabla w_{n}\right|^{2} \mathrm{~d} s \leq C .
$$

Obviously,

$$
\delta_{0}-\varepsilon_{n} \leq \delta_{n} \leq \delta_{0}<\min \left\{\delta_{1}, \delta_{2}, \vartheta_{j}\right\}
$$

Set

$$
t_{n}=\nabla V\left(\varepsilon_{n} y_{\varepsilon_{n}, j}\right)=\left(t_{n}^{1}, t_{n}^{2}, t_{n}^{3}\right) .
$$

Since $w_{n} \in W_{\text {loc }}^{2, q}\left(\mathbb{R}^{3}, \mathbb{C}^{4}\right)$ for $q \in[1,+\infty)$ (see Lemma 4.1) and $w_{n}$ is of class $C^{1}$, we can take the scalar product of

$$
D w_{n}+a \beta w_{n}+V\left(\varepsilon_{n} x\right) w_{n}-\frac{1}{8} \tilde{\chi}\left(\varepsilon_{n} x\right) V\left(\varepsilon_{n} x\right) \widetilde{\xi}\left(x,\left|w_{n}\right|\right) w_{n}=f_{\varepsilon_{n}}\left(x,\left|w_{n}\right|\right) w_{n},
$$

with

$$
t_{n} \cdot \nabla w_{n}:=\sum_{k=1}^{3} t_{n}^{k} \partial_{k} w_{n}
$$

and integrate on $B\left(y_{\varepsilon_{n}, j}, \delta_{n} \varepsilon_{n}^{-1}\right)$. Then similarly as that in (4.8) there holds $\varepsilon_{n} x \in B(0, \widetilde{R})$ and $\tilde{\chi}\left(\varepsilon_{n} x\right)=0$ for any $x \in B\left(y_{\varepsilon_{n}, j}, \delta_{n} \varepsilon_{n}^{-1}\right)$, which implies

$$
\operatorname{Re} \int_{B\left(y_{\varepsilon_{n}, j}, \delta_{n} \varepsilon_{n}^{-1}\right)}\left(D w_{n}+a \beta w_{n}+V\left(\varepsilon_{n} x\right) w_{n}-f_{\varepsilon_{n}}\left(x,\left|w_{n}\right|\right) w_{n}\right) \cdot\left(t_{n} \cdot \nabla w_{n}\right)=0 .
$$

Computing directly, by divergence theorem, we have

$$
\begin{aligned}
& \int_{B\left(y_{\varepsilon_{n}, j}, \delta_{n} \varepsilon_{n}^{-1}\right)} \operatorname{Re} p\left|w_{n}\right|^{p-2} w_{n} \cdot\left(t_{n} \cdot \nabla w_{n}\right) \\
& \quad=\int_{B\left(y_{\varepsilon_{n}, j}, \delta_{n} \varepsilon_{n}^{-1}\right)} \operatorname{div}\left(\left|w_{n}\right|^{p} t_{n}\right)=\int_{\partial B\left(y_{\varepsilon_{n}, j}, \delta_{n} \varepsilon_{n}^{-1}\right)}\left|w_{n}\right|^{p}\left(t_{n} * v\right),
\end{aligned}
$$

and

$$
\begin{aligned}
& \int_{B\left(y_{\varepsilon_{n}, j}, \delta_{n} \varepsilon_{n}^{-1}\right)} \operatorname{Re} \chi\left(\varepsilon_{n} x\right) p\left|w_{n}\right|^{p-2} w_{n} \cdot\left(t_{n} \cdot \nabla w_{n}\right)+\varepsilon_{n}\left(\nabla \chi\left(\varepsilon_{n} x\right)\left|w_{n}\right|^{p} * t_{n}\right) \\
& =\int_{B\left(y_{\varepsilon_{n}, j}, \delta_{n} \varepsilon_{n}^{-1}\right)} \operatorname{div}\left(\chi\left(\varepsilon_{n} x\right)\left|w_{n}\right|^{p} t_{n}\right)=\int_{\partial B\left(y_{\varepsilon_{n}, j}, \delta_{n} \varepsilon_{n}^{-1}\right)} \chi\left(\varepsilon_{n} x\right)\left|w_{n}\right|^{p}\left(t_{n} * v\right) .
\end{aligned}
$$


Moreover, by the definition of $g(x, t)$ and the boundedness of $t_{n}$ ( $V$ is of class $C^{1}$ and $\varepsilon_{n} y_{\varepsilon_{n}, j}$ is bounded),

$$
\begin{aligned}
& \left|\int_{B\left(y_{\varepsilon_{n}, j}, \delta_{n} \varepsilon_{n}^{-1}\right)} \chi\left(\varepsilon_{n} x\right) g\left(x,\left|w_{n}\right|\right) w_{n} \cdot\left(t_{n} \cdot \nabla w_{n}\right)\right| \\
& \quad \leq C \varepsilon_{n}^{4} \int_{B\left(y_{\varepsilon_{n}, j}, \delta_{n} \varepsilon_{n}^{-1}\right)}\left|w_{n}\right|\left|\nabla w_{n}\right|=O\left(\varepsilon_{n}^{4}\right) .
\end{aligned}
$$

Then the combination of the above three estimates leads us to the assertion that

$$
\begin{aligned}
& \operatorname{Re} \int_{B\left(y_{\varepsilon_{n}, j}, \delta_{n} \varepsilon_{n}^{-1}\right)} f_{\varepsilon_{n}}\left(x,\left|w_{n}\right|\right) w_{n} \cdot\left(t_{n} \cdot \nabla w_{n}\right)-\frac{\varepsilon_{n}}{p}\left(\nabla \chi\left(\varepsilon_{n} x\right)\left|w_{n}\right|^{p} * t_{n}\right) \\
& =\int_{\partial B\left(y_{\varepsilon_{n}, j}, \delta_{n} \varepsilon_{n}^{-1}\right)} \frac{1}{p}\left(1-\chi\left(\varepsilon_{n} x\right)\right)\left|w_{n}\right|^{p}\left(t_{n} * v\right)+O\left(\varepsilon_{n}^{4}\right) .
\end{aligned}
$$

To estimate the rest terms, similarly as that in Lemma 4.5 of [33], we have

$$
\begin{gathered}
2 \operatorname{Re} \int_{B\left(y_{\varepsilon_{n}, j}, \delta_{n} \varepsilon_{n}^{-1}\right)} a \beta w_{n} \cdot\left(t_{n} \cdot \nabla w_{n}\right)=\int_{\partial B\left(y_{\varepsilon_{n}, j}, \delta_{n} \varepsilon_{n}^{-1}\right)}\left(a \beta w_{n} \cdot w_{n}\right)\left(t_{n} * v\right), \\
\int_{B\left(y_{\varepsilon_{n}, j}, \delta_{n} \varepsilon_{n}^{-1}\right)} 2 \operatorname{Re} V\left(\varepsilon_{n} x\right) w_{n} \cdot\left(t_{n} \cdot \nabla w_{n}\right)+\varepsilon_{n}\left|w_{n}\right|^{2}\left(\nabla V\left(\varepsilon_{n} x\right) * t_{n}\right) \\
=\int_{\partial B\left(y_{\varepsilon_{n}, j}, \delta_{n} \varepsilon_{n}^{-1}\right)} V\left(\varepsilon_{n} x\right)\left|w_{n}\right|^{2}\left(t_{n} * v\right),
\end{gathered}
$$

and

$$
\begin{aligned}
& \operatorname{Re} \int_{B\left(y_{\varepsilon_{n}, j}, \delta_{n} \varepsilon_{n}^{-1}\right)} D w_{n} \cdot\left(t_{n} \cdot \nabla w_{n}\right) \\
& \quad=\frac{1}{2} \int_{\partial B\left(y_{\varepsilon_{n}, j}, \delta_{n} \varepsilon_{n}^{-1}\right)}\left(D w_{n} \cdot w_{n}\right)\left(t_{n} * v\right)-\left(\sum_{m=1}^{3} t_{n}^{m} \partial_{m} w_{n} \cdot i \sum_{k=1}^{3} v_{k} \alpha_{k} w_{n}\right),
\end{aligned}
$$

where $v=\left(v_{1}, v_{2}, v_{3}\right)$ is the outward unit vector of $\partial B\left(y_{\varepsilon_{n}, j}, \delta_{n} \varepsilon_{n}^{-1}\right)$. Then, from (4.15) to (4.18), it follows that

$$
\begin{aligned}
& \int_{B\left(y_{\varepsilon_{n}, j}, \delta_{n} \varepsilon_{n}^{-1}\right)} \frac{1}{2} \varepsilon_{n}\left|w_{n}\right|^{2}\left(\nabla V\left(\varepsilon_{n} x\right) * t_{n}\right)+\frac{\varepsilon_{n}}{p}\left(\nabla \chi\left(\varepsilon_{n} x\right)\left|w_{n}\right|^{p} * t_{n}\right) \\
& =\int_{\partial B\left(y_{\varepsilon_{n}, j}, \delta_{n} \varepsilon_{n}^{-1}\right)} \frac{1}{2}\left(a \beta w_{n} \cdot w_{n}\right)\left(t_{n} * v\right)+\frac{1}{2} V\left(\varepsilon_{n} x\right)\left|w_{n}\right|^{2}\left(t_{n} * v\right) \\
& \quad-\frac{1}{p}\left(1-\chi\left(\varepsilon_{n} x\right)\right)\left|w_{n}\right|^{p}\left(t_{n} * v\right)+\frac{1}{2}\left(D w_{n} \cdot w_{n}\right)\left(t_{n} * v\right) \\
& \quad-\frac{1}{2}\left(\sum_{m=1}^{3} t_{n}^{m} \partial_{m} w_{n} \cdot i \sum_{k=1}^{3} v_{k} \alpha_{k} w_{n}\right) \mathrm{d} s+O\left(\varepsilon_{n}^{4}\right) .
\end{aligned}
$$


Since $w_{n}\left(\cdot+y_{\varepsilon_{n}, j}\right) \rightarrow w_{j} \neq 0$ in $L_{\text {loc }}^{2}\left(\mathbb{R}^{3}, \mathbb{C}^{4}\right)$ (see Lemma 4.2), it follows from (4.11) and (4.14) that, for sufficiently large $n$

$$
\begin{aligned}
& \int_{B\left(y_{\varepsilon_{n}, j}, \delta_{n} \varepsilon_{n}^{-1}\right)} \varepsilon_{n}\left|w_{n}\right|^{2}\left(\nabla V\left(\varepsilon_{n} x\right) * t_{n}\right) \\
& \quad \geq \frac{\varepsilon_{n}}{2}\left|\nabla V\left(y_{j}\right)\right|^{2} \int_{B\left(0, R_{j}\right)}\left|w_{n}\left(\cdot+y_{\varepsilon_{n}, j}\right)\right|^{2} \\
& \quad \geq C \varepsilon_{n},
\end{aligned}
$$

with

$$
\int_{B\left(0, R_{j}\right)}\left|w_{j}\right|^{2} \geq \frac{1}{2} \int_{\mathbb{R}^{3}}\left|w_{j}\right|^{2}, \quad C=\frac{1}{4}\left|\nabla V\left(y_{j}\right)\right|^{2} \int_{\mathbb{R}^{3}}\left|w_{j}\right|^{2}>0 .
$$

Using the definition of $\chi$, for any $x \in \mathbb{R}^{3} \backslash \bar{\Lambda}_{\varepsilon_{n}}$, we have

$$
\nabla \chi\left(\varepsilon_{n} x\right) * t_{n}=\zeta^{\prime}\left(\operatorname{dist}\left(\varepsilon_{n} x, \Lambda\right)\right) \nabla \operatorname{dist}\left(\varepsilon_{n} x, \Lambda\right) * \nabla V\left(\varepsilon_{n} y_{\varepsilon_{n}, j}\right) .
$$

By (4.14), for any $x \in B\left(y_{\varepsilon_{n}}, j, \delta_{n} \varepsilon_{n}^{-1}\right) \backslash \bar{\Lambda}_{\varepsilon_{n}}$, there holds

$$
\varepsilon_{n} x \in B\left(\varepsilon_{n} y_{\varepsilon_{n}, j}, \delta_{1}\right) \backslash \bar{\Lambda} .
$$

This, together with (4.10), $\zeta^{\prime} \geq 0$ and the fact $\varepsilon_{n} y_{\varepsilon_{n}, j}=y_{j}+o_{\varepsilon_{n}}(1) \in \Lambda^{\delta_{1}}$ for large $n$, yields that

$$
\nabla \chi\left(\varepsilon_{n} x\right) * t_{n} \geq 0, \text { for } x \in B\left(y_{\varepsilon_{n}, j}, \delta_{n} \varepsilon_{n}^{-1}\right) \backslash \bar{\Lambda}_{\varepsilon_{n}},
$$

while, by the definition of $\chi$, we have $\nabla \chi\left(\varepsilon_{n} x\right) * t_{n}=0$ for $x \in \bar{\Lambda}_{\varepsilon_{n}}$. So

$$
\int_{B\left(y_{\varepsilon_{n}, j}, \delta_{n} \varepsilon_{n}^{-1}\right)} \varepsilon_{n}\left(\nabla \chi\left(\varepsilon_{n} x\right)\left|w_{n}\right|^{p} * t_{n}\right) \geq 0 .
$$

On the other hand, from (4.12), (4.13) and $\left|\delta_{n} \varepsilon_{n}^{-1}-\delta_{0} \varepsilon_{n}^{-1}\right| \leq 1$, it follows

$$
\begin{aligned}
& \int_{\partial B\left(y_{\varepsilon_{n}, j}, \delta_{n} \varepsilon_{n}^{-1}\right)}\left(D w_{n} \cdot w_{n}\right)\left(t_{n} * v\right)-\left(\sum_{m=1}^{3} t_{n}^{m} \partial_{m} w_{n} \cdot i \sum_{k=1}^{3} v_{k} \alpha_{k} w_{n}\right) \mathrm{d} s \\
& \leq C\left(\int_{\partial B\left(y_{\varepsilon_{n}, j}, \delta_{n} \varepsilon_{n}^{-1}\right)}\left|\nabla w_{n}\right|^{2} \mathrm{~d} s\right)^{\frac{1}{2}}\left(\int_{\partial B\left(y_{\varepsilon_{n}, j}, \delta_{n} \varepsilon_{n}^{-1}\right)}\left|w_{n}\right|^{2} \mathrm{~d} s\right)^{\frac{1}{2}} \leq C e^{-\frac{c}{\varepsilon_{n}}} .
\end{aligned}
$$

Similarly,

$$
\begin{aligned}
\int_{\partial B\left(y_{\varepsilon_{n}, j}, \delta_{n} \varepsilon_{n}^{-1}\right)} \frac{1}{2}\left(a \beta w_{n} \cdot w_{n}\right)\left(t_{n} * v\right) & +\frac{1}{2} V\left(\varepsilon_{n} x\right)\left|w_{n}\right|^{2}\left(t_{n} * v\right) \\
- & \frac{1}{p}\left(1-\chi\left(\varepsilon_{n} x\right)\right)\left|w_{n}\right|^{p}\left(t_{n} * v\right) \mathrm{d} s \leq C e^{-\frac{c}{\varepsilon_{n}}} .
\end{aligned}
$$

Then taking (4.20)-(4.23) into (4.19), we get a contradiction.

Lemma 4.5 For any $\delta>0$, there exist $c=c(\delta)>0$ and $C=C(\delta)>0$, such that when $\varepsilon$ is small, there holds

$$
\left|w_{\varepsilon}(x)\right| \leq C \exp \left(-c|x|^{\frac{2-\tau}{2}}\right), \quad \varepsilon x \in \mathbb{R}^{3} \backslash \mathcal{V}^{\delta}
$$


Proof First, since $\lim _{\varepsilon \rightarrow 0} \operatorname{dist}\left(\varepsilon y_{\varepsilon, j}, \mathcal{V}\right)=0, j=1, \ldots, m$, similarly as that in (4.7), for any $\delta>0$, there holds

$$
\lim _{\varepsilon \rightarrow 0}\left|w_{\varepsilon}(x)\right|=0 \text { uniformly for } x \in \mathbb{R}^{3} \backslash\left(\mathcal{V}^{\delta / 2}\right)_{\varepsilon} .
$$

Then by a similar method as that in Proposition 4.3, we can prove the existence of $c(\delta)>0$ and $C(\delta)>0$, such that

$$
\left|w_{\varepsilon}(x)\right| \leq C \exp (-c / \varepsilon) \quad \varepsilon x \in \overline{\mathcal{V}^{\delta}} \backslash \mathcal{V}^{\delta / 2}, \quad \varepsilon \in\left(0, \varepsilon_{0}\right) .
$$

Recalling that

$$
h_{\varepsilon}\left(x,\left|w_{\varepsilon}\right|\right)=-V_{\varepsilon}(x)+\frac{1}{8} \tilde{\chi}(\varepsilon x) V(\varepsilon x) \tilde{\xi}\left(x,\left|w_{\varepsilon}\right|\right)+f_{\varepsilon}\left(x,\left|w_{\varepsilon}\right|\right),
$$

by $\tilde{\xi} \in[0,2]$ and the definition of $f_{\varepsilon}$, we have

$$
\left|h_{\varepsilon}\left(x,\left|w_{\varepsilon}\right|\right)\right| \leq\left|V_{\varepsilon}(x)\right|+\phi(x), \quad \varepsilon x \in \mathbb{R}^{3} \backslash B(0, \widetilde{R}) .
$$

This, together with $\left(\mathrm{V}_{1}\right)$ and the choice of $\widetilde{R}$ such that $2 \gamma /\left(1+\widetilde{R}^{\tau}\right) \leq a$, yields that

$$
a^{2}-\left(h_{\varepsilon}\left(x,\left|w_{\varepsilon}\right|\right)\right)^{2} \geq \frac{a \gamma}{1+|\varepsilon x|^{\tau}}, \quad \varepsilon x \in \mathbb{R}^{3} \backslash B(0, \widetilde{R}), \quad \varepsilon \in\left(0, \varepsilon_{0}\right),
$$

while, for $\varepsilon x \in B(0, \widetilde{R}) \backslash \mathcal{V}^{\delta / 2}$, it follows from (2.4) and (4.24) that

$$
a^{2}-\left(h_{\varepsilon}\left(x,\left|w_{\varepsilon}\right|\right)\right)^{2} \geq \theta^{\prime}>0, \quad \varepsilon \in\left(0, \varepsilon_{0}\right) .
$$

Since $w_{\varepsilon}$ satisfies (4.4): $\Delta\left|w_{\varepsilon}\right| \geq a^{2}\left|w_{\varepsilon}\right|-\left(h_{\varepsilon}\left(x,\left|w_{\varepsilon}\right|\right)\right)^{2}\left|w_{\varepsilon}\right|$, and hence, for constant $\theta^{\prime \prime}>0$ and $\varepsilon \in\left(0, \varepsilon_{0}\right)$, there weakly holds

$$
-\Delta\left|w_{\varepsilon}\right|+\frac{\theta^{\prime \prime}}{1+|\varepsilon x|^{\tau}}\left|w_{\varepsilon}\right| \leq 0, \varepsilon x \in \mathbb{R}^{3} \backslash \mathcal{V}^{\delta / 2} .
$$

Let $u_{\varepsilon}(x):=w_{\varepsilon}\left(\frac{x}{\varepsilon}\right)$. When $\varepsilon_{0}$ is small and $\varepsilon \in\left(0, \varepsilon_{0}\right)$, there holds

$$
-\varepsilon^{2} \Delta\left|u_{\varepsilon}\right|+\frac{\theta^{\prime \prime}}{1+|x|^{\tau}}\left|u_{\varepsilon}\right| \leq 0, \quad x \in \mathbb{R}^{3} \backslash \mathcal{V}^{\delta / 2} \text {. }
$$

Note that there exists $c^{\prime}(\delta)>0$, such that $\psi_{\varepsilon}:=\exp \left(-c^{\prime}|x / \varepsilon|^{\frac{2-\tau}{2}}\right)$ is a sub-solution

$$
-\varepsilon^{2} \Delta \psi_{\varepsilon}+\frac{\theta^{\prime \prime}}{1+|x|^{\tau}} \psi_{\varepsilon} \geq 0, \quad x \in \mathbb{R}^{3} \backslash \mathcal{V}^{\delta / 2} .
$$

Then by the maximum principle and (4.25), there exist $c=c(\delta)>0$ and $C=C(\delta)>0$, such that when $\varepsilon_{0}$ is small and $\varepsilon \in\left(0, \varepsilon_{0}\right)$,

$$
\left|u_{\varepsilon}(x)\right| \leq C \exp \left(-c|x / \varepsilon|^{\frac{2-\tau}{2}}\right), x \in \mathbb{R}^{3} \backslash \mathcal{V}^{\delta},
$$

and the conclusion follows obviously.

Proof of Theorem 1.1 Since $\mathcal{V}$ is a compact subset of $\Lambda$,

$$
\operatorname{dist}(\mathcal{V}, \partial \Lambda)>0 \text {. }
$$

Fixing a positive $\delta<\operatorname{dist}(\mathcal{V}, \partial \Lambda)$, by Lemma 4.5 , we have

$$
\left|w_{\varepsilon}(x)\right| \leq C \exp \left(-c|x|^{\frac{2-\tau}{2}}\right), \quad x \in \mathbb{R}^{3} \backslash \Lambda_{\varepsilon}, \quad \varepsilon \in\left(0, \varepsilon_{0}\right) .
$$


Making $\varepsilon_{0}$ smaller if necessary, for $\varepsilon \in\left(0, \varepsilon_{0}\right)$, there hold

$$
\left|w_{\varepsilon}(x)\right|,\left|w_{\varepsilon}(x)\right|^{p-2} \leq \phi(x), \quad x \in \mathbb{R}^{3} \backslash \Lambda_{\varepsilon},
$$

and hence $\tilde{\chi}(\varepsilon x) \tilde{\xi}\left(x,\left|w_{\varepsilon}\right|\right)=0, f_{\varepsilon}\left(x,\left|w_{\varepsilon}\right|\right)=\left|w_{\varepsilon}\right|^{p-2}$. Thus, $w_{\varepsilon}$ is actually a solution of $\left(\mathrm{D}_{\varepsilon}\right)$ and $u_{\varepsilon}(x)=w_{\varepsilon}(x / \varepsilon)$ is a solution of (D). Additionally, from (4.26) the decay property of $u_{\varepsilon}$ naturally follows.

\section{Some extensions}

\subsection{Multiplicity result}

In this section, we first prove Theorem 1.2 by the following abstract critical point theorem (see [4] Theorem 5.2 and [5] Theorem 4.6 for the proof).

Theorem 5.1 Let $\left(X,\|\cdot\|_{X}\right)$ be a separable reflexive Banach space with orthogonal decomposition $X=X^{-} \oplus X^{+}, I \in C^{1}(X, \mathbb{R})$ be even, satisfy the Palais-Smale condition. Assume the following assumptions hold:

(i) There exist $r>0$ and $\rho>0$, such that

$$
\inf _{u \in X^{+},\|u\|_{X}=r} I(u) \geq \rho .
$$

(ii) There exist an $N$-dimensional subspace $X_{N}=\operatorname{span}\left\{e_{1}, \ldots, e_{N}\right\} \subset X^{+}$and an $R_{N}>$ $r$ such that

$$
\sup _{u \in X^{-} \oplus X_{N}} I(u)<\infty \quad \text { and } \sup _{u \in X^{-} \oplus X_{N},\|u\|_{X}>R_{N}} I(u)<\inf _{u \in X^{+},\|u\|_{X} \leq r} I(u) .
$$

(iii) Let $\mathcal{S}$ be a countable dense subset of $\left(X^{-}\right)^{*}$ (the dual space of $\left.X^{-}\right), \mathcal{P}$ be the family of seminorms on $X$ consisting of all seminorms:

$$
p_{s}: X \rightarrow \mathbb{R} ; \quad p_{s}(u)=\left|s\left(u^{-}\right)\right|+\left\|u^{+}\right\|_{X}, u=u^{+}+u^{-} \in X, s \in \mathcal{S} .
$$

If we denote the induced topology by $\left(X, \mathcal{T}_{\mathcal{P}}\right)$ and denote the weak ${ }^{*}$ topology on $X^{*}$ by $\left(X^{*}, \mathcal{T}_{w^{*}}\right)$, there hold, for any $c \in \mathbb{R}, I_{c}:=\{u \in X: I(u) \geq c\}$ is $\mathcal{T}_{\mathcal{P}}$-closed and $I^{\prime}:\left(I_{c}, \mathcal{T}_{\mathcal{P}}\right) \rightarrow\left(X^{*}, \mathcal{T}_{w^{*}}\right)$ is continuous.

Then I has at least $N$ pairs of critical points $\pm u_{j}$ with

$$
I\left(u_{j}\right) \in\left[\rho, \sup _{u \in X^{-} \oplus X_{N}} I(u)\right], \quad j=1, \ldots, N .
$$

By (3.1), Lemma 3.3 and a similar argument as that in Lemma 3.2 , we know that for any positive integer $N>0$, there exists $\varepsilon_{N}>0$ such that when $\varepsilon \in\left(0, \varepsilon_{N}\right), \Phi_{\varepsilon}$ satisfies the hypotheses of Theorem 5.1. And hence we can obtain $N$ pairs of solutions $\pm w_{\varepsilon, j}$, $j=1,2, \ldots, N$, corresponding to $\Phi_{\varepsilon}$ with $0<\rho_{0} \leq \Phi_{\varepsilon}\left(w_{\varepsilon, j}\right) \leq C, j=1,2, \ldots, N$. Then by the same procedures of proof as that in Sect. 4, we can show that these solutions possess the exponential decay and are in fact solutions of the original problem. 


\subsection{Competing potentials}

We remark that our method works also for the case of competing potentials. Let us consider the following system with competing potentials $V$ and $K$,

$$
-i \varepsilon \sum_{k=1}^{3} \alpha_{k} \partial_{k} u+a \beta u+V(x) u=K(x)|u|^{p-2} u, \quad u \in H^{1}\left(\mathbb{R}^{3}, \mathbb{C}^{4}\right),
$$

where $p \in(2,3), V$ and $K$ satisfy the following assumptions.

$\left(\mathrm{V}_{1}\right) \quad V \in C^{1}\left(\mathbb{R}^{3}, \mathbb{R}\right)$, for constants $\tau \in(0,2)$ and $\gamma \in(0,+\infty)$

$$
a-|V(x)| \geq \frac{\gamma}{1+|x|^{\tau}} .
$$

$\left(\mathrm{K}_{1}\right) K \in C^{1}\left(\mathbb{R}^{3}, \mathbb{R}\right)$ and $0<k_{1} \leq K(x) \leq k_{2}(1+|x|)^{\tau^{\prime}}$ for any $x \in \mathbb{R}^{3}$ with constants $k_{2}>0$ and $\tau^{\prime}>0$

(VK) There is a bounded domain $\Lambda \subset \mathbb{R}^{3}$ with smooth boundary $\partial \Lambda$ such that

$$
\begin{gathered}
\vec{n}(x) * \nabla V(x)>0 \text { for any } x \in \partial \Lambda, \\
\nabla K(x) * \nabla V(x)<0 \text { for any } x \in \partial \Lambda .
\end{gathered}
$$

where $\vec{n}(x)$ denotes the unit outward normal vector to $\partial \Lambda$ at $x$.

Denote for $\delta>0$ small $U(\delta)=\{x \in \Lambda: \operatorname{dist}(x, \partial \Lambda)>\delta\}$. Then there is $\delta_{1}>0$ such that $\sup _{\Lambda^{\delta_{1} \backslash U\left(\delta_{1}\right)}} \nabla K(x) * \nabla V(x)<0$. And we will have the following result.

Theorem 5.2 For $p \in(2,3)$, suppose that assumptions $\left(\mathrm{V}_{1}\right),\left(\mathrm{K}_{1}\right)$ and $(\mathrm{VK})$ hold. Then for any positive integer $N$, there exists $\varepsilon_{N}>0$ such that if $0<\varepsilon<\varepsilon_{N}$, Eq. (5.1) has at least $N$ pairs of solutions $\pm u_{\varepsilon, j}, j=1,2, \ldots, N$, satisfying that, for any $\delta_{1}>\delta>0$, there exist $c=c(\delta, N)>0$ and $C=C(\delta, N)>0$ such that

$$
\left|u_{\varepsilon, j}(x)\right| \leq C \exp \left(-c\left(\frac{\operatorname{dist}(x, U(\delta))}{\varepsilon}\right)^{\frac{2-\tau}{2}}\right), \quad 1 \leq j \leq N .
$$

Clearly, the equivalent problem is given by

$$
-i \alpha \cdot \nabla u+a \beta u+V(\varepsilon x) u=K(\varepsilon x)|u|^{p-2} u .
$$

Making $\delta_{1}$ smaller if necessary such that there also holds (2.3), then we define the penalized functional $Q_{\varepsilon}$ by the same way as that in Sect. 2 with

$$
\phi(x):=\frac{1}{1+|x|^{\tau^{\prime}+4}} .
$$

Let $g(x, t)=\min \left\{t^{p-2}, \phi(x)\right\}$. We modified the nonlinearity by

$$
f_{\varepsilon}(x, t)=(1-\chi(\varepsilon x)) K(\varepsilon x) t^{p-2}+\chi(\varepsilon x) K(\varepsilon x) g(x, t), \quad F_{\varepsilon}(x, t)=\int_{0}^{t} f_{\varepsilon}(x, s) s \mathrm{~d} s,
$$

and give the modified functional

$$
\Phi_{\varepsilon}(u)=\frac{1}{2}\left(\left\|u^{+}\right\|^{2}-\left\|u^{-}\right\|^{2}\right)+\frac{1}{2} \int_{\mathbb{R}^{3}} V(\varepsilon x)|u|^{2}-Q_{\varepsilon}(u)-\int_{\mathbb{R}^{3}} F_{\varepsilon}(x,|u|) .
$$

Then the rest of the proof is almost the same as above, and the main difference is in Lemma 4.4 in particular the proof of (4.15). 
Lemma 5.3 For any $1 \leq l \leq m, \lim _{\varepsilon \rightarrow 0} \operatorname{dist}\left(\varepsilon y_{\varepsilon, l}, U\left(\delta_{1}\right)\right)=0$.

Arguing indirectly, we assume that there exists $\varepsilon_{n}>0$ such that $\lim _{n \rightarrow \infty} \varepsilon_{n}=0$ and

$$
\lim _{n \rightarrow \infty} \operatorname{dist}\left(\varepsilon_{n} y_{\varepsilon_{n}, l}, U\left(\delta_{1}\right)\right)>0 .
$$

However, by using the divergence theorem and the assumption in (VK)

$$
\nabla K(x) * \nabla V(x)<0 \text { for any } x \in \Lambda \backslash U\left(\delta_{1}\right),
$$

it is not difficult to show that the lemma is true. And for other details in the proof, we leave them to the readers.

Acknowledgements Research was supported by the Specialized Fund for the Doctoral Program of Higher Education of China, NSFC 11771324 and a Simons Collaboration Grant.

\section{Appendix}

Proof of Lemma 4.2 Obviously, $w_{\varepsilon} \rightarrow w_{0}$ in $E$ with $w_{0}$ a solution of

$$
-i \alpha \cdot \nabla u+a \beta u+V(0) u=|u|^{p-2} u
$$

We define $w_{\varepsilon, 0}:=w_{\varepsilon}-w_{0}$, and there holds

$$
\left\|w_{\varepsilon, 0}\right\|^{2}=\left\|w_{\varepsilon}\right\|^{2}-\left\|w_{0}\right\|^{2}+o_{\varepsilon}(1)
$$

It is easy to see, as $\varepsilon \rightarrow 0$,

$$
\begin{aligned}
& \int_{\mathbb{R}^{3}}(V(\varepsilon x)-V(0)) w_{0} \cdot \psi=o_{\varepsilon}(1)\|\psi\|, \\
& \int_{\mathbb{R}^{3}} \tilde{\chi}(\varepsilon x) V(\varepsilon x) \tilde{\xi}\left(x,\left|w_{\varepsilon}\right|\right) w_{0} \cdot \psi=o_{\varepsilon}(1)\|\psi\| .
\end{aligned}
$$

uniformly holds for $\psi \in E$. Similarly,

$$
\int_{\mathbb{R}^{3}}\left(f_{\varepsilon}\left(x,\left|w_{0}\right|\right) w_{0}-\left|w_{0}\right|^{p-2} w_{0}\right) \cdot \psi=o_{\varepsilon}(1)\|\psi\|, \quad \varepsilon \rightarrow 0
$$

uniformly holds for $\psi \in E$. And by a standard argument, we know that, as $\varepsilon \rightarrow 0$,

$$
f_{\varepsilon}\left(x,\left|w_{\varepsilon}\right|\right) w_{\varepsilon}-f_{\varepsilon}\left(x,\left|w_{\varepsilon, 0}\right|\right) w_{\varepsilon, 0}-f_{\varepsilon}\left(x,\left|w_{0}\right|\right) w_{0} \rightarrow 0 \text { in } E^{*},
$$

where $E^{*}$ is the dual of $E$. Therefore,

$$
f_{\varepsilon}\left(x,\left|w_{\varepsilon}\right|\right) w_{\varepsilon}-f_{\varepsilon}\left(x,\left|w_{\varepsilon, 0}\right|\right) w_{\varepsilon, 0}-\left|w_{0}\right|^{p-2} w_{0} \rightarrow 0 \text { in } E^{*}
$$


Then computing directly, using (5.3) and (5.4), we deduce that

$$
\begin{aligned}
\int_{\mathbb{R}^{3}} & \left(H_{0} w_{\varepsilon, 0}+V(\varepsilon x) w_{\varepsilon, 0}-\frac{1}{8} \tilde{\chi}(\varepsilon x) V(\varepsilon x) \tilde{\xi}\left(x,\left|w_{\varepsilon}\right|\right) w_{\varepsilon, 0}-f_{\varepsilon}\left(x,\left|w_{\varepsilon, 0}\right|\right) w_{\varepsilon, 0}\right) \cdot \psi \\
= & \int_{\mathbb{R}^{3}}\left(H_{0} w_{\varepsilon}+V(\varepsilon x) w_{\varepsilon}-\frac{1}{8} \tilde{\chi}(\varepsilon x) V(\varepsilon x) \tilde{\xi}\left(x,\left|w_{\varepsilon}\right|\right) w_{\varepsilon}-f_{\varepsilon}\left(x,\left|w_{\varepsilon}\right|\right) w_{\varepsilon}\right) \cdot \psi \\
& -\int_{\mathbb{R}^{3}}\left(H_{0} w_{0}+V(0) w_{0}-\left|w_{0}\right|^{p-2} w_{0}\right) \cdot \psi+(V(\varepsilon x)-V(0)) w_{0} \cdot \psi \\
& +\int_{\mathbb{R}^{3}}\left(f_{\varepsilon}\left(x,\left|w_{\varepsilon}\right|\right) w_{\varepsilon}-f_{\varepsilon}\left(x,\left|w_{\varepsilon, 0}\right|\right) w_{\varepsilon, 0}-\left|w_{0}\right|^{p-2} w_{0}\right) \cdot \psi \\
& +\int_{\mathbb{R}^{3}} \frac{1}{8} \tilde{\chi}(\varepsilon x) V(\varepsilon x) \tilde{\xi}\left(x,\left|w_{\varepsilon}\right|\right) w_{0} \cdot \psi=o_{\varepsilon}(1)\|\psi\|,
\end{aligned}
$$

uniformly holds for $\psi \in E$ as $\varepsilon \rightarrow 0$. If there holds

$$
\lim _{\varepsilon \rightarrow 0} \sup _{z \in \mathbb{R}^{3}} \int_{B(z, 1)}\left|w_{\varepsilon, 0}\right|^{2}=0,
$$

then by Lions' lemma [28], we know $\left|w_{\varepsilon, 0}\right|_{q} \rightarrow 0, q \in(2,3)$ as $\varepsilon \rightarrow 0$ and the proof is done.

Otherwise, there exists subsequence, which we still denote by $\{\varepsilon\}$, such that

$$
\underline{\lim _{\varepsilon \rightarrow 0}} \sup _{z \in \mathbb{R}^{3}} \int_{B(z, 1)}\left|w_{\varepsilon, 0}\right|^{2} \geq \tau_{0}>0 .
$$

Let $\left\{y_{\varepsilon, 1}\right\} \subset \mathbb{R}^{3}$ be points such that

$$
\int_{B\left(y_{\varepsilon, 1}, 1\right)}\left|w_{\varepsilon, 0}\right|^{2} \geq \frac{\tau_{0}}{2}
$$

Since $w_{\varepsilon, 0} \rightarrow 0$ in $E$, there holds $\left|y_{\varepsilon, 1}\right| \rightarrow \infty$. We claim that $\varepsilon y_{\varepsilon, 1} \rightarrow y_{1} \in \Lambda^{\delta_{1}}$ as $\varepsilon \rightarrow 0$. Setting $\widetilde{w}_{\varepsilon, 0}(x)=w_{\varepsilon, 0}\left(x+y_{\varepsilon, 1}\right)$, we have $\widetilde{w}_{\varepsilon, 0} \rightarrow w_{1}$ in $E$ with $w_{1} \neq 0$. Moreover from (5.5), $\widetilde{w}_{\varepsilon, 0}$ satisfies

$$
\begin{aligned}
& -i \alpha \cdot \nabla \widetilde{w}_{\varepsilon, 0}+a \beta \widetilde{w}_{\varepsilon, 0}+V\left(\varepsilon x+\varepsilon y_{\varepsilon, 1}\right) \widetilde{w}_{\varepsilon, 0}-f_{\varepsilon}\left(x+y_{\varepsilon, 1},\left|\widetilde{w}_{\varepsilon, 0}\right|\right) \widetilde{w}_{\varepsilon, 0} \\
& -\frac{1}{8} \tilde{\chi}\left(\varepsilon x+\varepsilon y_{\varepsilon, 1}\right) V\left(\varepsilon x+\varepsilon y_{\varepsilon, 1}\right) \tilde{\xi}\left(x+y_{\varepsilon, 1},\left|w_{\varepsilon}\left(\cdot+y_{\varepsilon, 1}\right)\right|\right) \widetilde{w}_{\varepsilon, 0}=o_{\varepsilon}(1) \text { in } E^{*} .
\end{aligned}
$$

Testing the equation by $w_{1}^{+}-w_{1}^{-}$and assuming by contradiction that $\left|\varepsilon y_{\varepsilon, 1}\right| \rightarrow \infty$ or $\varepsilon y_{\varepsilon, 1} \rightarrow y_{1} \notin \Lambda^{\delta_{1}}$ as $\varepsilon \rightarrow 0$, we have

$$
\begin{aligned}
o_{\varepsilon}(1) & \left\|w_{1}\right\| \\
= & \operatorname{Re} \int_{\mathbb{R}^{3}}\left(H_{0} \widetilde{w}_{\varepsilon, 0}+V\left(\varepsilon x+\varepsilon y_{\varepsilon, 1}\right) \widetilde{w}_{\varepsilon, 0}-f_{\varepsilon}\left(x+y_{\varepsilon, 1},\left|\widetilde{w}_{\varepsilon, 0}\right|\right) \widetilde{w}_{\varepsilon, 0}\right) \cdot\left(w_{1}^{+}-w_{1}^{-}\right) \\
& -\frac{1}{8} \widetilde{\chi}\left(\varepsilon x+\varepsilon y_{\varepsilon, 1}\right) V\left(\varepsilon x+\varepsilon y_{\varepsilon, 1}\right) \widetilde{\xi}\left(x+y_{\varepsilon, 1},\left|w_{\varepsilon}\left(\cdot+y_{\varepsilon, 1}\right)\right|\right) \widetilde{w}_{\varepsilon, 0} \cdot\left(w_{1}^{+}-w_{1}^{-}\right) \\
= & \operatorname{Re} \int_{\mathbb{R}^{3}}\left(H_{0} w_{1}+V_{1} w_{1}-\frac{1}{8} \widetilde{\chi}_{1} V_{1} \widetilde{\xi}_{\varepsilon, 1} w_{1}\right) \cdot\left(w_{1}^{+}-w_{1}^{-}\right)+o_{\varepsilon}(1) \\
= & \left\|w_{1}\right\|^{2}+\int_{\mathbb{R}^{3}} V_{1}\left(\left|w_{1}^{+}\right|^{2}-\left|w_{1}^{-}\right|^{2}\right)-\frac{1}{8} \widetilde{\chi}_{1} V_{1} \widetilde{\xi}_{\varepsilon, 1}\left(\left|w_{1}^{+}\right|^{2}-\left|w_{1}^{-}\right|^{2}\right)+o_{\varepsilon}(1) .
\end{aligned}
$$


Here $V_{1}=\lim _{\varepsilon \rightarrow 0} V\left(\varepsilon y_{\varepsilon, 1}\right), \widetilde{\chi}_{1}=\lim _{\varepsilon \rightarrow 0} \tilde{\chi}\left(\varepsilon y_{\varepsilon}\right)$ and $\widetilde{\xi}_{\varepsilon, 1}=\widetilde{\xi}\left(x+y_{\varepsilon, 1},\left|w_{\varepsilon}\left(\cdot+y_{\varepsilon, 1}\right)\right|\right) ;$ moreover, we have used the facts that

$$
\left|\int_{\mathbb{R}^{3}} \chi(\varepsilon x) g(x, u) u \cdot h\right| \leq C \varepsilon^{4}\|u\|\|h\|, \text { uniformly for } u, h \in E,
$$

and that when $\left|\varepsilon y_{\varepsilon, 1}\right| \rightarrow \infty$ or $\varepsilon y_{\varepsilon, 1} \rightarrow y_{1} \notin \Lambda^{\delta_{1}}$,

$$
\int_{\mathbb{R}^{3}}\left(1-\chi\left(\varepsilon x+\varepsilon y_{\varepsilon, 1}\right)\right)\left|\widetilde{w}_{\varepsilon, 0}\right|^{p-2} \widetilde{w}_{\varepsilon, 0} \cdot h=o_{\varepsilon}(1), \quad h \in E .
$$

Let $\varepsilon \rightarrow 0$ in the above inequality. By $\tilde{\chi}_{1} \in[0,1], V_{1} \in[-a, a]$ and $\widetilde{\xi}_{\varepsilon, 1} \in[0,2]$, we deduce

$$
0 \geq\left\|w_{1}\right\|^{2}-a \int_{\mathbb{R}^{3}}\left|w_{1}\right|^{2}
$$

while, since $\sigma_{e}\left(H_{0}\right)=(-\infty,-a] \cup[a,+\infty),\left\|w_{1}\right\|^{2}-a\left|w_{1}\right|_{2}=0$ implies that $w_{1}=0$, which is a contradiction. Thus there holds $\varepsilon y_{\varepsilon, 1} \rightarrow y_{1} \in \Lambda^{\delta_{1}}$ as $\varepsilon \rightarrow 0$, and consequently

$$
\tilde{\chi}_{1}=\lim _{\varepsilon \rightarrow 0} \tilde{\chi}\left(\varepsilon y_{\varepsilon, 1}\right)=\tilde{\chi}\left(y_{1}\right)=\tilde{\zeta}\left(\left|y_{1}\right|-\widetilde{R}\right)=0 .
$$

Therefore, if we use an arbitrary $\psi \in C_{0}^{\infty}\left(\mathbb{R}^{3}, \mathbb{C}^{4}\right)$ to test (5.6), we will see that $w_{1}$ is a nontrivial solution of

$$
-i \alpha \cdot \nabla u+a \beta u+V\left(y_{1}\right) u=\left(1-\chi\left(y_{1}\right)\right)|u|^{p-2} u .
$$

Moreover, taking the scalar product with $w_{1}^{+}-w_{1}^{-}$on both sides and using the facts $y_{1} \in \Lambda^{\delta_{1}}$ and $1-\chi\left(y_{1}\right) \in(0,1]$, it is easy to see that

$$
\left\|w_{1}\right\| \geq \varrho>0 .
$$

We define $w_{\varepsilon, 1}:=w_{\varepsilon, 0}-w_{1}\left(\cdot-y_{\varepsilon, 1}\right)$ and

$$
v_{\varepsilon, 1}:=w_{\varepsilon, 1}\left(\cdot+y_{\varepsilon, 1}\right)=w_{\varepsilon, 0}\left(\cdot+y_{\varepsilon, 1}\right)-w_{1}=\widetilde{w}_{\varepsilon, 0}-w_{1} .
$$

Since $\widetilde{w}_{\varepsilon, 0} \rightarrow w_{1}$ weakly in $E$, there holds

$$
\left\|w_{\varepsilon, 1}\right\|^{2}=\left\|v_{\varepsilon, 1}\right\|^{2}=\left\|\tilde{w}_{\varepsilon, 0}\right\|^{2}-\left\|w_{1}\right\|^{2}+o_{\varepsilon}(1)=\left\|w_{\varepsilon, 0}\right\|^{2}-\left\|w_{1}\right\|^{2}+o_{\varepsilon}(1) .
$$

Noting that $\varepsilon y_{\varepsilon, 1} \rightarrow y_{1} \in \Lambda^{\delta_{1}} \subset B(0, \widetilde{R})$, similarly as above, we have

$$
\begin{aligned}
& \int_{\mathbb{R}^{3}}\left(V\left(\varepsilon x+\varepsilon y_{\varepsilon, 1}\right)-V\left(y_{1}\right)\right) w_{1} \cdot \psi=o_{\varepsilon}(1)\|\psi\|, \\
& \int_{\mathbb{R}^{3}} \tilde{\chi}\left(\varepsilon x+\varepsilon y_{\varepsilon, 1}\right) V\left(\varepsilon x+\varepsilon y_{\varepsilon, 1}\right) \tilde{\xi}\left(x+y_{\varepsilon, 1},\left|w_{\varepsilon}\left(\cdot+y_{\varepsilon, 1}\right)\right|\right) w_{1} \cdot \psi=o_{\varepsilon}(1)\|\psi\|,
\end{aligned}
$$

as $\varepsilon \rightarrow 0$ uniformly holds for $\psi \in E$. And there holds, as $\varepsilon \rightarrow 0$,

$$
f_{\varepsilon}\left(x+y_{\varepsilon, 1},\left|\widetilde{w}_{\varepsilon, 0}\right|\right) \widetilde{w}_{\varepsilon, 0}-f_{\varepsilon}\left(x+y_{\varepsilon, 1},\left|v_{\varepsilon, 1}\right|\right) v_{\varepsilon, 1}-f\left(y_{1},\left|w_{1}\right|\right) w_{1} \rightarrow 0 \text { in } E^{*} .
$$


Since $\widetilde{w}_{\varepsilon, 0}$ and $w_{1}$ satisfy (5.6) and (5.7), respectively, similarly as that in (5.5), we can deduce that

$$
\begin{aligned}
\int_{\mathbb{R}^{3}} & \left(H_{0} w_{\varepsilon, 1}+V(\varepsilon x) w_{\varepsilon, 1}-\frac{1}{8} \tilde{\chi}(\varepsilon x) V(\varepsilon x) \tilde{\xi}\left(x,\left|w_{\varepsilon}\right|\right) w_{\varepsilon, 1}-f_{\varepsilon}\left(x,\left|w_{\varepsilon, 1}\right|\right) w_{\varepsilon, 1}\right) \cdot \psi \\
= & \int_{\mathbb{R}^{3}}\left(H_{0} v_{\varepsilon, 1}+V\left(\varepsilon x+\varepsilon y_{\varepsilon, 1}\right) v_{\varepsilon, 1}-f_{\varepsilon}\left(x+y_{\varepsilon, 1},\left|v_{\varepsilon, 1}\right|\right) v_{\varepsilon, 1}\right. \\
& \left.-\frac{1}{8} \tilde{\chi}\left(\varepsilon x+\varepsilon y_{\varepsilon, 1}\right) V\left(\varepsilon x+\varepsilon y_{\varepsilon, 1}\right) \tilde{\xi}\left(x+y_{\varepsilon, 1},\left|w_{\varepsilon}\left(\cdot+y_{\varepsilon, 1}\right)\right|\right) v_{\varepsilon, 1}\right) \cdot \psi_{\varepsilon} \\
= & o_{\varepsilon}(1)\|\psi\|,
\end{aligned}
$$

uniformly holds for $\psi \in E$ as $\varepsilon \rightarrow 0$, where $\psi_{\varepsilon}=\psi\left(\cdot+y_{\varepsilon, j}^{1}\right)$. If there holds

$$
\lim _{\varepsilon \rightarrow 0} \sup _{z \in \mathbb{R}^{3}} \int_{B(z, 1)}\left|w_{\varepsilon, 1}\right|^{2}=0,
$$

then by Lions' lemma [28], $\left|w_{\varepsilon, 1}\right|_{q} \rightarrow 0, q \in(2,3)$ as $\varepsilon \rightarrow 0$ and we are finished with the proof.

Otherwise, we repeat the above procedure and obtain a sequence $\left\{y_{\varepsilon, 2}\right\}$ such that, as $\varepsilon \rightarrow 0, \varepsilon y_{\varepsilon, 2} \rightarrow y_{2} \in \Lambda^{\delta_{1}},\left|y_{\varepsilon, 2}\right| \rightarrow \infty,\left|y_{\varepsilon, j}-y_{\varepsilon, j^{\prime}}\right| \rightarrow \infty, 1 \leq j \neq j^{\prime} \leq 2$ and

$$
\widetilde{w}_{\varepsilon, 1}=w_{\varepsilon, 1}\left(\cdot+y_{\varepsilon, 2}\right) \rightarrow w_{2},
$$

where $\left\|w_{2}\right\| \geq \varrho$ is a nontrivial solution of

$$
-i \alpha \cdot \nabla u+a \beta u+V\left(y_{2}\right) u=\left(1-\chi\left(y_{2}\right)\right)|u|^{p-2} u .
$$

Moreover, for $w_{\varepsilon, 2}:=w_{\varepsilon, 1}-w_{2}\left(\cdot-y_{\varepsilon, 2}\right)$, there holds [see (5.2) and (5.8)]

$$
\left\|w_{\varepsilon, 2}\right\|^{2}=\left\|w_{\varepsilon, 1}\right\|^{2}-\left\|w_{2}\right\|^{2}+o_{\varepsilon}(1)=\left\|w_{\varepsilon}\right\|^{2}-\sum_{j=0}^{2}\left\|w_{j}\right\|^{2}+o_{\varepsilon}(1) .
$$

We observe that the iteration procedure has to stop in a finite number of steps $m$, because of $\left\|w_{\varepsilon}\right\|^{2} \leq C_{R_{0}}$ (see Theorem 3.4) and $\left\|w_{j}\right\| \geq \varrho$ for each $j$. Thus we complete the proof.

\section{References}

1. Ambrosetti, A., Malchiodi, A.: Perturbation Methods and Semilinear Elliptic Problems on $\mathbb{R}^{N}$, Progress in Mathematics, vol. 240. Birkhäuser, Basel (2006)

2. Ambrosetti, A., Felli, V., Malchiodi, A.: Ground states of nonlinear Schrödinger equations with potentials vanishing at infinity. J. Eur. Math. Soc. (JEMS) 7(1), 117-144 (2005)

3. Balabane, M., Cazenave, T., Douady, A., Merle, F.: Existence of excited states for a nonlinear Dirac field. Commun. Math. Phys. 119(1), 153-176 (1988)

4. Bartsch, T., Ding, Y.H.: Solutions of nonlinear Dirac equations. J. Differ. Equ. 226(1), 210-249 (2006)

5. Bartsch, T., Ding, Y.H.: Deformation theorems on non-metrizable vector spaces and applications to critical point theory. Math. Nachr. 279(12), 1267-1288 (2006)

6. Byeon, J., Wang, Z.-Q.: Standing waves with a critical frequency for nonlinear Schrödinger equations. Arch. Ration. Mech. Anal. 165(4), 295-316 (2002)

7. Byeon, J., Wang, Z.-Q.: Standing waves with a critical frequency for nonlinear Schrödinger equations. II. Calc. Var. Partial Differ. Equ. 18(2), 207-219 (2003)

8. Cazenave, T., Vazquez, L.: Existence of localized solutions for a classical nonlinear Dirac field. Commun. Math. Phys. 105(1), 35-47 (1986) 
9. Cerami, G., Devillanova, G., Solimini, S.: Infinitely many bound states for some nonlinear scalar field equations. Calc. Var. Partial Differ. Equ. 23(2), 139-168 (2005)

10. Chen, S.W., Wang, Z.-Q.: Localized nodal solutions of higher topological type for semiclassical nonlinear Schrödinger equations. Calc. Var. Partial Differ. Equ. 56(1), 1 (2017)

11. Dautray, R., Lions, J.L.: Mathematical Analysis and Numerical Methods for Science and Technology, vol. 3. Springer, Berlin (1990)

12. Del Pino, M., Felmer, P.: Semi-classical states for nonlinear Schröinger equations. J. Funct. Anal. 149(1), 245-265 (1997)

13. Del Pino, M., Felmer, P.: Multi-peak bound states for nonlinear Schröinger equations. Ann. Inst. H. Poincaré Anal. Non Lináire 15(2), 127-149 (1998)

14. Ding, Y.H.: Variational Methods for Strongly Indefinite Problems, vol. 7. World Scientific Publishing, Singapore (2007)

15. Ding, Y.H.: Semi-classical ground states concentrating on the nonlinear potential for a Dirac equation. J. Differ. Equ. 249(5), 1015-1034 (2010)

16. Ding, Y.H., Lee, C., Ruf, B.: On semiclassical states of a nonlinear Dirac equation. Proc. R. Soc. Edinb. Sect. A 143(4), 765-790 (2013)

17. Ding, Y.H., Ruf, B.: Solutions of a nonlinear Dirac equation with external fields. Arch. Ration. Mech. Anal. 190(1), 57-82 (2008)

18. Ding, Y.H., Ruf, B.: Existence and concentration of semiclassical solutions for Dirac equations with critical nonlinearities. SIAM J. Math. Anal. 44(6), 3755-3785 (2012)

19. Ding, Y.H., Wei, J.C.: Stationary states of nonlinear Dirac equations with general potentials. J. Math. Phys. 20(8), 1007-1032 (2008)

20. Ding, Y.H., Wei, J.C., Xu, T.: Existence and concentration of semi-classical solutions for a nonlinear Maxwell-Dirac system. J. Math. Phys. 54(6), 061505 (2013)

21. Ding, Y.H., Xu, T.: Localized concentration of semi-classical states for nonlinear Dirac equations. Arch. Ration. Mech. Anal. 216(2), 415-447 (2015)

22. Esteban, M.J., Séré, E.: Stationary states of the nonlinear Dirac equation: a variational approach. Commun. Math. Phys. 171(2), 323-350 (1995)

23. Esteban, M.J., Lewin, M., Séré, E.: Variational methods in relativistic quantum mechanics. Bull. Am. Math. Soc. (N.S.) 45(4), 535-593 (2008)

24. Finkelstein, R., LeLevier, R., Ruderman, M.: Nonlinear spinor fields. Phys. Rev. 83(2), 326-332 (1951)

25. Finkelstein, R., Fronsdal, C., Kaus, P.: Nonlinear spinor field. Phys. Rev. 103(5), 1571-1579 (1956)

26. Gilbarg, D., Trudinger, N.: Elliptic Partial Differential Equations of Second Order. Springer, Berlin (1997)

27. Kang, X., Wei, J.C.: On interacting bumps of semi-classical states of nonlinear Schrödinger equations. Adv. Differ. Equ. 5(7-9), 899-928 (2000)

28. Lions, P.L.: The concentration-compactness principle in the calculus of variations. The locally compact case, parts 1 and 2. In: Annales de l'Institut Henri Poincaré Anual. Non Linéair, vol. 1, pp. 109-145, 223-283 (1984)

29. Merle, F.: Existence of stationary states for nonlinear Dirac equations. J. Differ. Equ. 74(1), 50-68 (1988)

30. Moroz, V., Van Schaftingen, S.: Semiclassical stationary states for nonlinear Schrödinger equations with fast decaying potentials. Calc. Var. Partial Differ. Equ. 37(1), 1-27 (2010)

31. Pankov, A.: On decay of solutions to nonlinear Schrödinger equations. Proc. Am. Math. Soc. 136(7), 2565-2570 (2008)

32. Rabinowitz, P.H.: On a class of nonlinear Schrödinger equations. Z. Angew. Math. Phys. 43(2), 270-292 (1992)

33. Wang, Z.-Q., Zhang, X.: An infinite sequence of localized semiclassical bound states for nonlinear Dirac equations. Calc. Var. Partial Differ. Equ. 57(2), 30 (2018). (Art. 56)

Publisher's Note Springer Nature remains neutral with regard to jurisdictional claims in published maps and institutional affiliations. 